N $63 \quad 18338$

\title{
563205
}

Technical Report No. 32-417

\section{Probe Measurements of the Discharge in an Operating Electron Bombardment Engine}

William B. Strickfaden

Kenneth L. Geiler
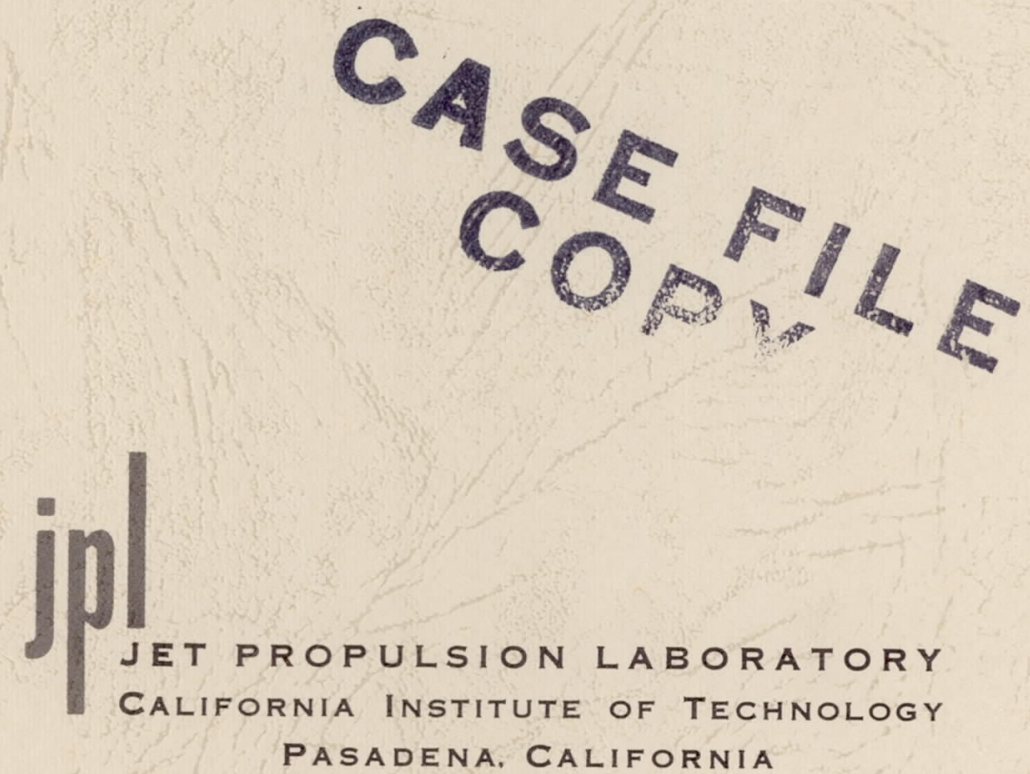

April 19, 1963 

Technical Report No. 32-417

\title{
Probe Measurements of the Discharge in an Operating Electron Bombardment Engine
}

\author{
William B. Strickfaden \\ Kenneth L. Geiler
}

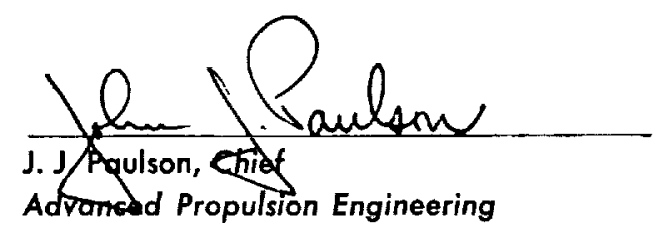

JET PROPULSION LABORATORY CALIFORNIA INSTITUTE OF TECHNOLOGY

PASADENA, CALIFORNIA

April 19, 1963 


\section{Copyright (C) 1963}

Jet Propulsion Laboratory California Institute of Technology

Prepared Under Contract No. NAS 7-100

National Aeronautics \& Space Administration 


\section{CONTENTS}

I. Introduction . . . . . . . . . . . . . . . . . 1

II. Experimental Setup . . . . . . . . . . . . . . . 2

III. Typical Results . . . . . . . . . . . . . . . . . . . . 4

IV. Analysis of LPC . . . . . . . . . . . . . . . . . . . . 7

v. Results . . . . . . . . . . . . . . . . . . . . . . . 9

VI. Conclusions . . . . . . . . . . . . . . . . . . 21

References . . . . . . . . . . . . . . 22

\section{FIGURES}

1. Circuit diagram of engine and probe . . . . . . . . . . . . . 2

2. View of experimental setup . . . . . . . . . . . . . . . . 3

3. Movable probe . . . . . . . . . . . . . . . . . . . . 3

4. Variable-area probe . . . . . . . . . . . . . . . . . . . 3

5. Typical semilogarithmic LPC showing variation in probe trace with arc volfage . . . . . . . . . . . . . . . . . . 4

6. Typical semilogarithmic LPC from variable area probe . . . . . . . 5

7. Typical radial semilogarithmic LPCs . . . . . . . . . . . . . 5

8. Typical LPC obłained on oscilloscope . . . . . . . . . . . . . . . . 6

9. Typical LPC obtained on oscilloscope showing oscillations . . . . . . . 6

10. Typical LPC from $x-y$ recorder

11. Corrected and uncorrected semilogarithmic LPC . . . . . . . . . 8

12. Positions at which measurements were taken . . . . . . . . . . . . 9

13. Radial plot of electron density and energy variations (no accelerating polential applied) . . . . . . . . . . . . . . 10

14. Radial plot of electron density and energy variations (accelerating potential applied) . . . . . . . . . . . . . . . 11

15. Radial plot of electron density and energy variations (no accelerating porential applied) . 


\section{FIGURES (Cont'd)}

16. Radial plot of electron density and energy variations (accelerating potential applied) . . . . . . . . . . . . . . . 13

17. Radial plot of electron energy and density variations near grids (no accelerating potential applied) . . . . . . . . . . . . . . 14

18. Radial plot of electron energy and density variations near grids (accelerating potential applied) . . . . . . . . . . . . . . . 15

19. Axial plot of electron energy and density variations (no accelerating potential applied) . . , . . . , . , . . . 16

20. Axial plot of electron energy and density variations (accelerafing potential applied) . . , , , , , , . . . , 17

21. Approximation of the mercury ionization cross section . . . . . . . . 18

22. Ionization coefficient for Maxwell electrons $\quad$. . . . . . . . . . . . . 19

23. Ionization rate curve (no accelerating potential applied) . . . . . . 19

24. Ionization rate curve (accelerating potential applied) . . . . . . . . 20 


\begin{abstract}
Current-voltage characteristics have been obtained from Langmuir probes at various positions inside a Kaufman-type ion motor. Data are presented for the engine in static (no beam, but plasma) and operating (160-ma beam) conditions. The analysis of the probe data indicates that the electrons in the plasma possess a non-Maxwellian distribution of energies. To obtain plasma properties from the probe curves, the non-Maxwellian energy distribution is assumed to consist of a primary monoenergetic group of high-energy electrons plus the usual Maxwell distribution. Working from this assumption the probe curves are analyzed for primary and Maxwell energies and densities, and the data for radial positions inside the engine are presented. It is shown from the density curves that the primary electrons are the principal source of ion production. The beam current as calculated from probe data near the exit grids agrees to the correct order of magnitude with the measured value.
\end{abstract}

\title{
I. INTRODUCTION
}

The possibilities of the Kaufman-type electron bombardment ion source (Ref. 1) for use as a thrust device for deep-space applications is well documented (Ref. 2). Even though the engine in its present state achieves reasonable efficiencies, its operation is not thoroughly understood. It has been studied externally; the variation of beam current, propellant utilization efficiency, etc., have been measured as the various external operating parameters such as arc voltage and magnetic field have been changed. For a deeper understanding of the source operation, it is necessary to probe the interior of the engine, where the plasma is being formed. Only in this way can a theoretical model be correlated with the actual situation. Then, if a proper model can be found, a calculation of the efficiencies and beam properties can be made from the operating parameters, and it will be possible to attempt to improve the engine operation for use as a thrust device.
This paper deals with an experimental program set up to investigate the properties of the plasma inside an operating Kaufman-type engine. From the results of this investigation it is hoped that a theoretical model can be developed which will sufficiently explain plasma formation so that future engines of this type can be built with predictable characteristics.

Owing to their small size and ability to distinguish plasma properties at different points, Langmuir probes (Ref. 3) were used to probe the inside of the engine. These probes are simple to operate and need a minimum amount of circuitry, advantages not to be overlooked when the engine is operated under flight-simulated conditions during which the whole device is floating at a potential of about $5 \mathrm{kv}$. 


\section{EXPERIMENTAL SETUP}

For this investigation an ion engine of the Kaufman type was purchased from Goodrich-High Voltage Astronautics, Inc. ${ }^{1}$ A schematic diagram of the engine and associated power supplies is given in Fig. 1.

The propellant, mercury, enters the arc or discharge chamber as a gas through a distributor in the rear of the engine. A cylindrical, nickel-matrix oxide, indirectly heated cathode is positioned along the axis of the engine. A cylindrical anode surrounds the cathode and is attached to, but electrically isolated from, the engine housing. The accelerator electrodes consist of two 0.060 -in. molybdenum plates through which a matrix of 0.187 -in. holes has been drilled in a close-pitch hexagonal array. One

${ }^{1}$ Now Ion Physics Corp., a subsidiary of High Voltage Engineering Corp., Burlington, Mass. of the electrodes is attached to and is electrically com-

mon with the engine housing which forms the arc chamber. The second electrode is mounted downstream of the first on three ceramic insulators. A solenoidal magnet is wound around the entire housing to provide field divergence downstream of the accelerating electrodes. A decelerating electrode is not employed.

During engine operation, the housing, cathode sheath, magnet, and propellant feed system are electrically common and maintained at high positive potential, while the outer accelerating electrode is biased negatively, typically -1000 to $-1500 \mathrm{v}$. The anode remains positive with respect to engine housing by the arc or discharge potential. The cathode temperature is varied to achieve a desired arc current for any selected arc voltage, thus ensuring emission-limited cathode operation.

The engine was installed in a 3 - by 7 - $\mathrm{ft}$ vacuum chamber capable of being evacuated to $10^{-7} \mathrm{~mm} \mathrm{Hg}$ (Fig. 2).

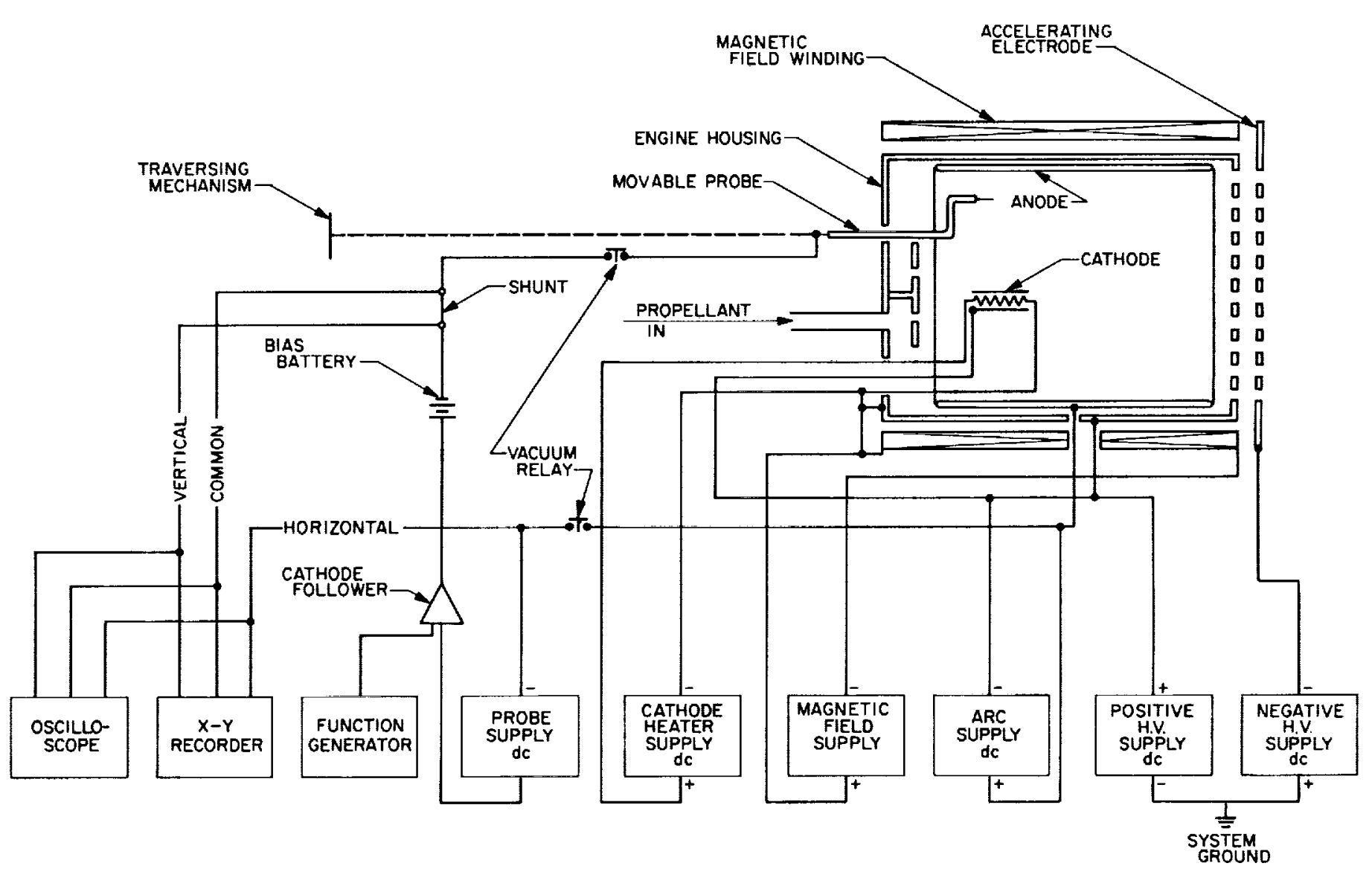

Fig. 1. Circuit diagram of engine and probe 
A cryogenically cooled collector was positioned 30 in. downstream of the engine-accelerating electrode. The collector is a two-section type, providing beam current measurements and suppression of secondary electrons.

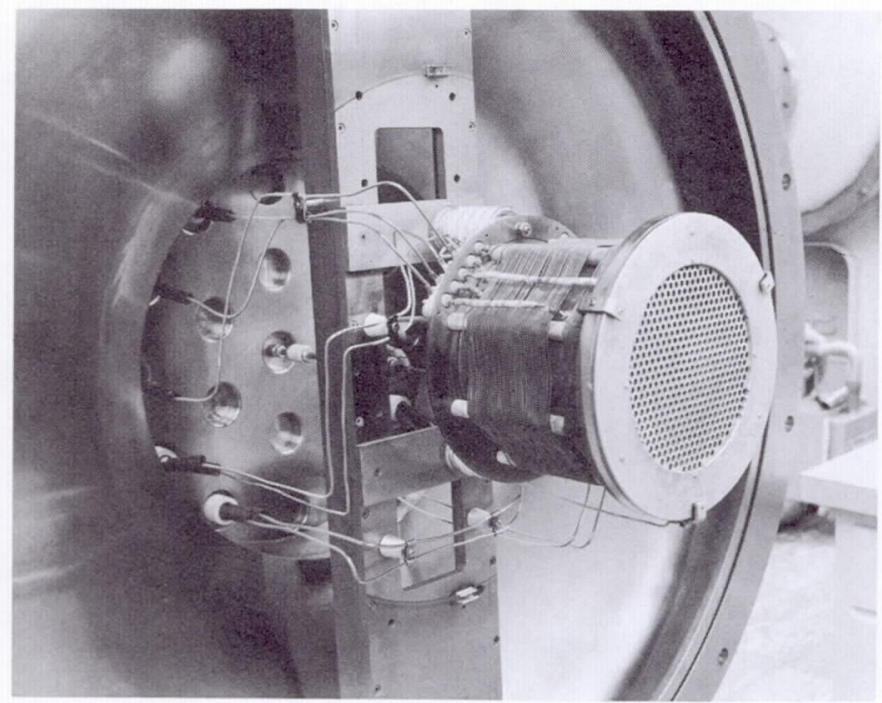

Fig. 2. View of experimental setup

A movable Langmuir probe (Fig. 3) was constructed by encapsulating a 0.020 -in.-diameter pure tungsten wire within a $96 \%$ fused silica sheath packed with finely powdered boron nitride. The tungsten wire was allowed to protrude $0.300 \mathrm{in}$. from the end of the sheath.

The probe was inserted into the plasma from the rear of the engine through a boron nitride seal and connected to a traversing and radial positioning mechanism located outside the vacuum tank. This mechanism was designed and constructed to provide probe position information to a tolerance of $\pm 0.015 \mathrm{in}$. in both the axial and radial directions.

Probe instrumentation, which consisted of a 0 - to 250 vdc power supply, variable frequency function generator, cathode follower amplifier, $x-y$ plotter with an input impedance of 1 megohm, oscilloscope, bias batteries, and 100 -ohm shunt, was electrically connected together as illustrated in Fig. 1. The instrumentation was isolated from ground potential and coupled to the ion engine and probe through two high-voltage vacuum relays, thus providing instrumentation decoupling capability without disturbing engine operation. All probe voltage information was referenced to anode potential.
A second Langmuir probe of variable area (Fig. 4) was constructed, for reasons discussed later in this paper. This probe consisted of a 0.090-in.-diameter pure tungsten rod sheathed in a boron nitride insulator. One end of the tungsten rod was attached to a solenoid which, when actuated, could vary the length of the rod protruding from the insulator. In the retracted position the rod protruded $0.050 \mathrm{in}$. from the insulator; in the extended position it protruded $0.350 \mathrm{in}$. The probe was inserted into the plasma from the rear of the engine. No traversing mechanism was employed on the second probe.

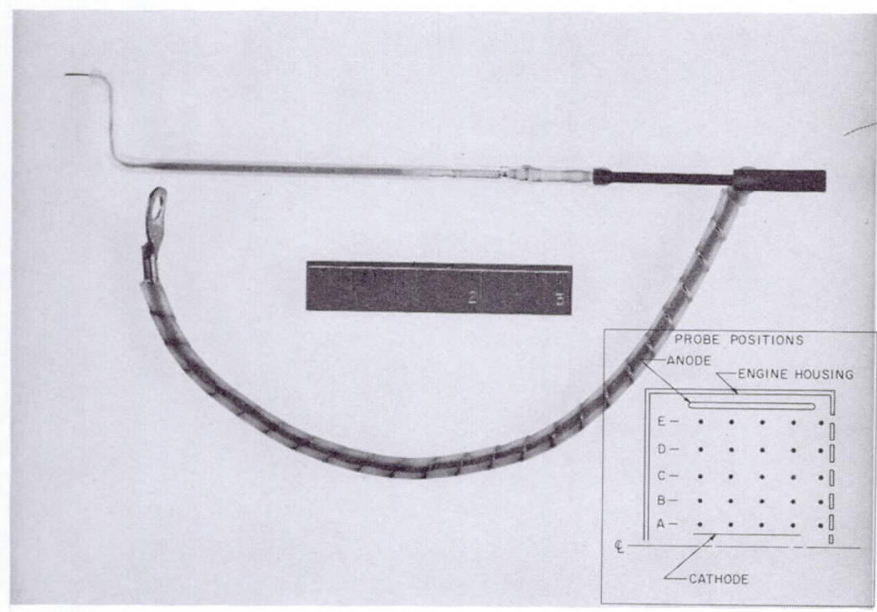

Fig. 3. Movable probe. Insert shows approximate probe positioning points inside engine

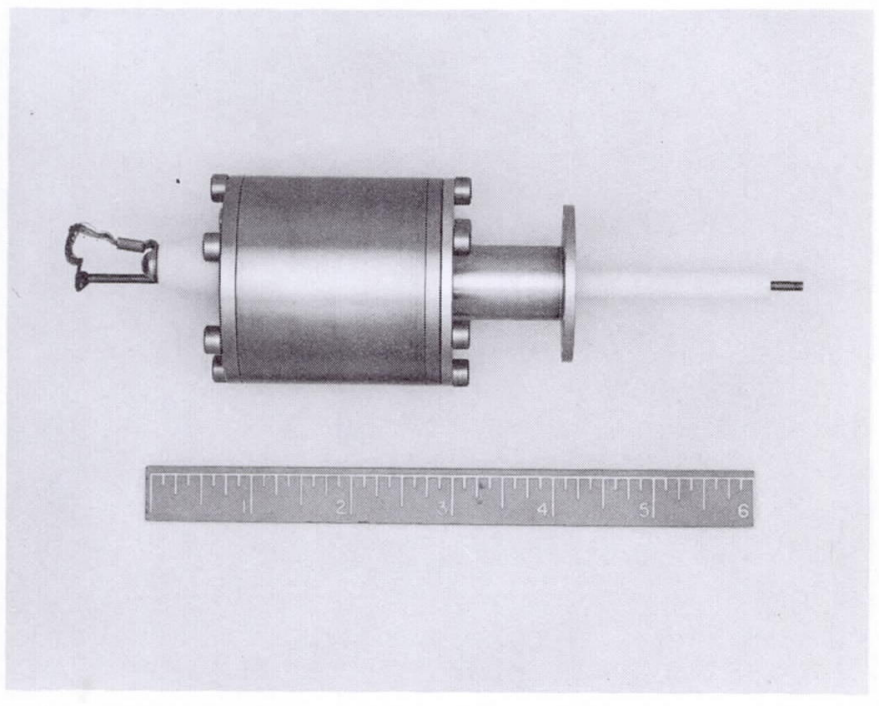

Fig. 4. Variable-area probe 


\section{TYPICAL RESULTS}

The analysis of a Langmuir probe characteristic (LPC) is simple if the electrons in the plasma are distributed according to the Maxwell-Boltzmann law. In this case a plot of the LPC on semilogarithmic graph paper will yield a straight line.

Quite early in this program many LPCs were obtained which did not plot as straight lines. Sometimes the deviation was small, sometimes large. In most cases the upper portion of the LPC was linear, the deviation from linearity not occurring until the probe was some 5 to $10 \mathrm{v}$ below plasma potential. In such cases, the question arises as to whether the deviations are due to an actual nonMaxwellian energy distribution law, or to some external cause such as an improper circuitry or a probe surface contamination, or to one of the many other phenomena evoked by experimenters using Langmuir probes. A definite answer to the question is hard to give, but from a series of independent experiments (some to be described herein) it appears that the anomalous LPCs are a result of a non-Maxwellian distribution law for the electrons in the plasma. It will also become apparent that the deviation from Maxwellian may be quite important in determining the operation of the source. A similar non-Maxwellian distribution has been reported by other workers, notably Medicus (Ref. 4), who studied a fireball discharge in neon.

Figure 5 presents a typical semilogarithmic plot of an LPC taken with the movable probe. Note the deviation from a straight line at negative voltage values. This type of behavior is typical of most of the LPCs taken under various operating conditions inside the engine. However, if the arc voltage is low enough, as shown in Fig. 5, the LPC follows a straight line for three decades of the current.

It is known that for some plasmas an anomalous characteristic such as that shown in Fig. 5 is found when the plasma creeps up the insulator of the probe with increasing negative voltages. To show that the anomalous LPCs do not occur from some probe-insulator-plasma interaction, the variable-area probe described previously was used. Figure 6 illustrates the results. The curve labeled "extended" is the LPC obtained by the probe in the extended position; any probe-insulator interaction should be present in this curve. The curve labeled "difference" is the difference between the two LPCs obtained by the probe in the extended and retracted positions; any insulator effect should not appear in the "difference" curve. A comparison of the "extended" and "difference" curves shows no difference, thus ruling out any effect of insulator-plasma interaction on the probe traces.

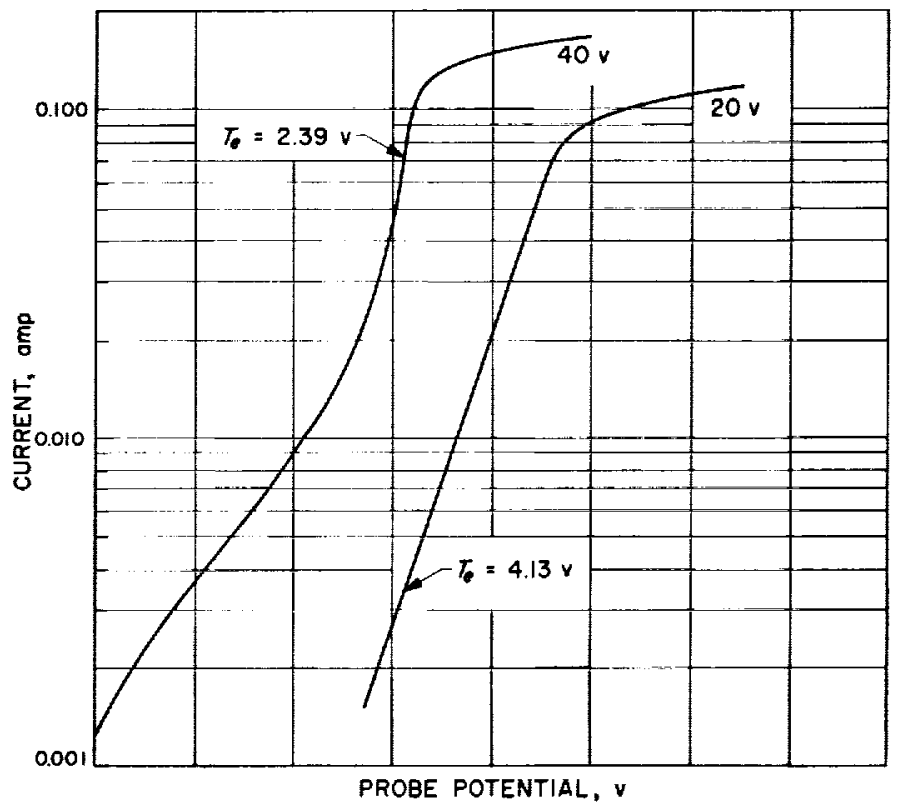

Fig. 5. Typical semilogarithmic LPC showing variation in probe trace with arc voltage

$\begin{array}{ll}\text { are voltage-noted } & \text { magnetic field-20 gauss } 16 \text { ampl } \\ \text { arc currenl-2 amp } & \text { flowrate-1.7 gm/hr }\end{array}$

To show that the anomalous LPCs do not result from the formation of oxides or amalgams or other contamination of the probe surface, the voltage on the probe was driven to high positive values. By sweeping the voltage on the probe at $0.01 \mathrm{cps}$, electron currents large enough to heat the probe to incandescence could be maintained for some tens of seconds. Heating by electron bombardment should raise the surface temperature of the probe high enough to boil off any contaminants.

It was found that LPCs taken with a probe immediately after cleaning in the above manner were identical to those taken when the probe was not heated to incandescence. The variable-area probe was not heated to incandescence.

From the above discussion, it is seen that the anomalous LPCs are probably due to an actual non-Maxwellian 
energy distribution for the electrons. Since the LPC at any voltage $V$ is an integral of the energy distribution taken from the energy $e V$ to infinity, an excess of highenergy electrons in the distribution would yield an LPC of the type observed. This interpretation is made throughout the remainder of this paper. Quantitative evaluation of the probe curves is made in Section IV.

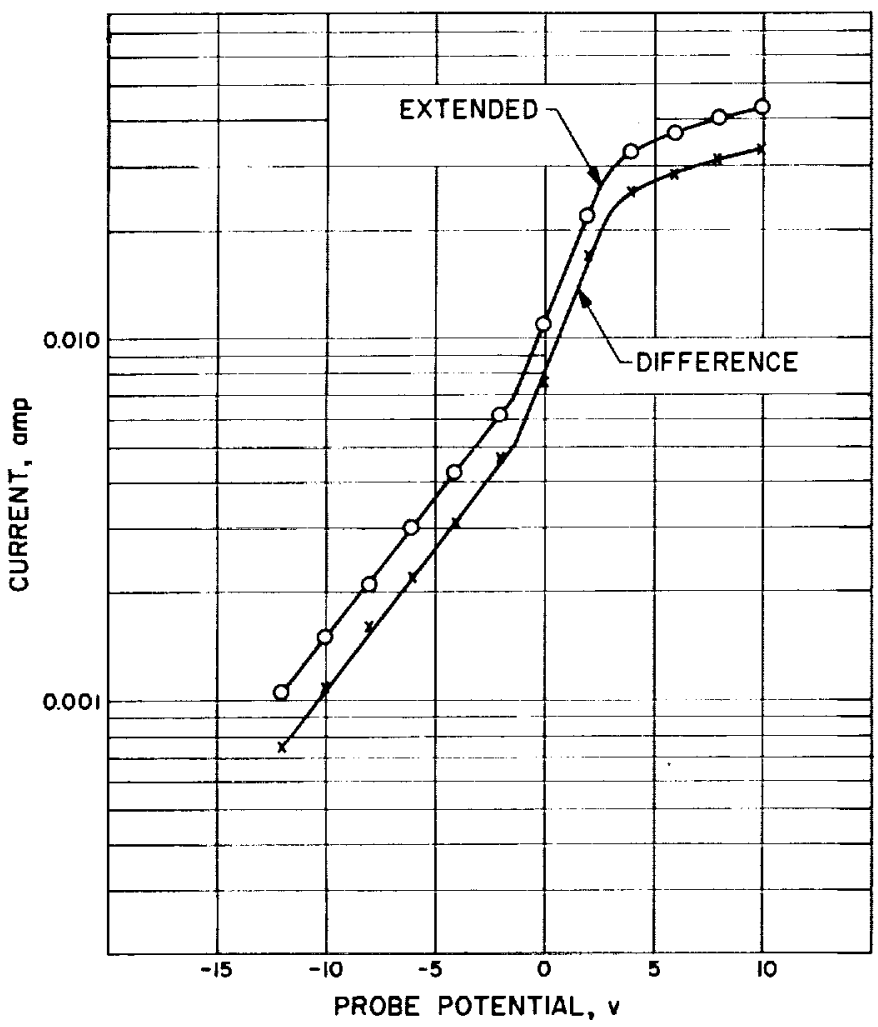

Fig. 6. Typical semilogarithmic LPC from variable-area probe

arc voltage-20 $\mathrm{V}$

arc current-0.85 amp

magnetic field-10 gauss ( 3 amp)

flowrate-1.7 gm $/ \mathrm{hr}$

Figure 7 shows five LPCs taken at different radial positions in the engine. (Engine operating parameters are noted in the caption.) Observe the gradual decrease in electron saturation current and general straightening of the curves near the anode. The saturation current is an approximate measure of the density of electrons which, as expected, decreases radially. This type of behavior is typical of all the LPCs obtained under many different operating conditions and was observed very near the grids when accelerating voltage was on or off.

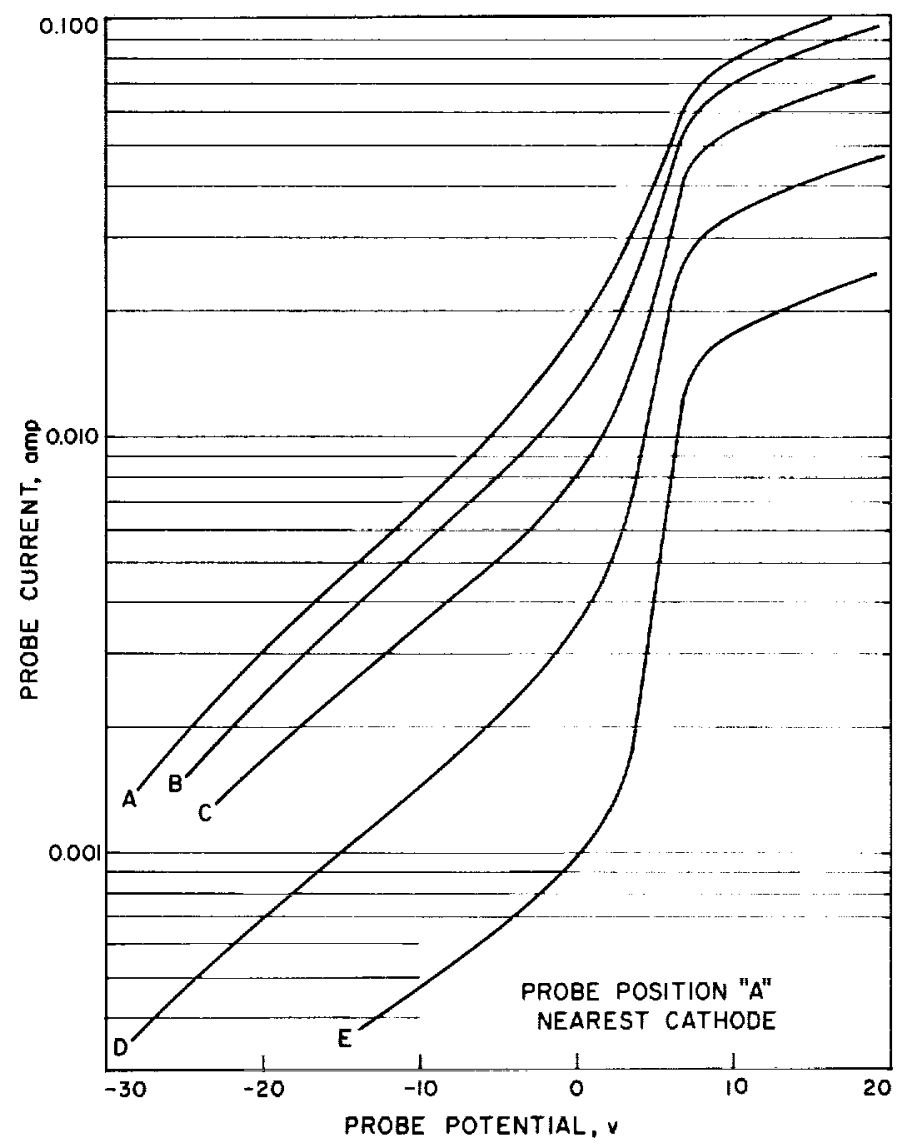

Fig. 7. Typical radial semilogarithmic LPCs

arc voltage $-50 \mathrm{v}$ magnetic field-14 gauss (4 amp)

arc current-2 amp flowrate- $1.7 \mathrm{gm} / \mathrm{hr}$

Under some operating conditions severe "hash" in the saturated electron current portion of the LPCs was observed when the characteristic was viewed on an oscilloscope. This phenomenon, which was interpreted as some sort of plasma oscillation, could be observed by coupling the oscilloscope to any portion of the engine. Figures 8 and 9 illustrate the magnitude of these oscillations. The only difference between the two pictures is the magnetic field intensity. Figure 8 was taken at 20 gauss; Fig. 9 at 25 gauss. These strong oscillations were always present above a certain critical field strength and may play an important role in engine operation. Since the LPCs to be used for analysis were taken with an $x-y$ recorder, no meaningful traces could be obtained when the strong oscillations were present. Therefore, it was not possible to obtain any information on the plasma when the magnetic field was above about 20 gauss. 


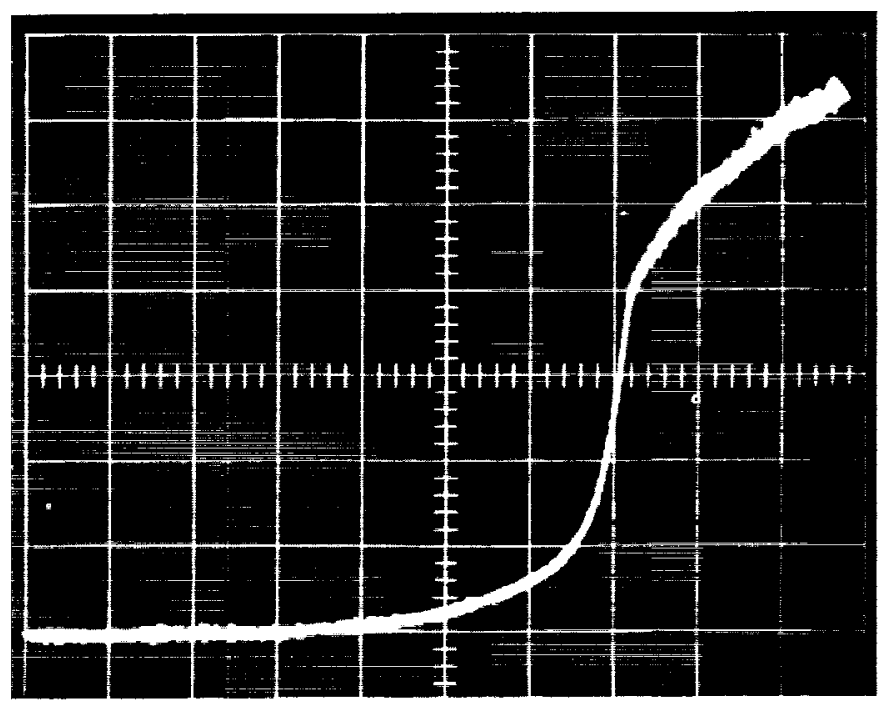

Fig. 8. Typical LPC obtained on oscilloscope

are voltage $-40 \mathrm{v}$

arc current-2 amp magnetic field-20 gauss (6 amp)

flowrate-1.7 gm/hr

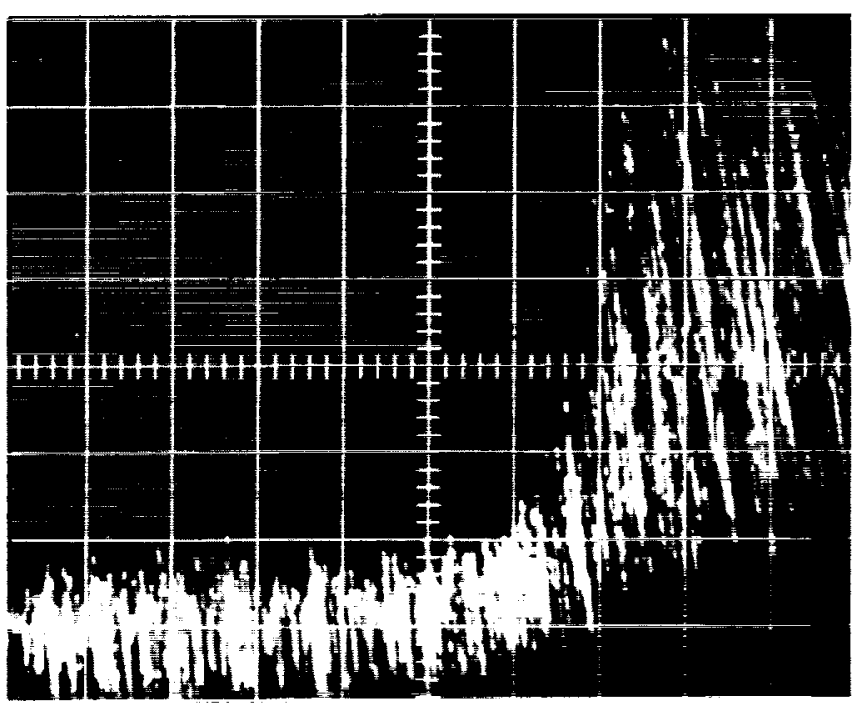

Fig. 9. Typical LPC obtained on oscilloscope showing oscillations

arc voltage $-40 \mathrm{v}$

orc current-2 amp magnetic fleld-25 gauss (9 amp)

flowrate-1.7 gm/hr 


\section{ANALYSIS OF LPC}

In order to obtain useful information on the plasma, a method of breaking down the LPC into an energy distribution for the electrons was devised. Before detailing this method, some general criteria are presented showing the applicability of Langmuir probes to investigations of plasma of the kind found in the Kaufman engine.

1. For the probe to act like an infinite planar probe, the sheath thickness should be small in comparison to the size of the probe. For a current density of $0.004 \mathrm{amp} / \mathrm{cm}^{2}$ and a voltage of $21.5 \mathrm{v}$, the sheath will be 9.8 by $10^{-3} \mathrm{~cm}$, as calculated by the space charge equation, which is much smaller than the diameter of any of the cylindrical probes used.

2. Elementary probe theory assumes that there are very few collisions in the sheath. This means that the sheath must be small in comparison to the mean free path of the electrons. In the Kaufmann engine, particle densities are of the order of $10^{11}$ particles/ cc, which gives a mean free path of several meters -much larger than the sheath indeed.

3. Since the probe is collecting electrons in the magnetic field of the engine, the effect of the magnetic field upon collection must either be corrected for or must be small enough to be neglected. If the cyclotron radius of the electrons is large compared to the probe dimensions, the effect of the magnetic field will be small. The cyclotron radius for a 5-ev electron in a 20 -gauss field is $0.3 \mathrm{~cm}$, which is large compared to the probe diameter of $0.05 \mathrm{~cm}$.

From the above data it is clear that the usual infinite planar probe theory will be applicable.

When the plasma electrons possess a Maxwell distribution of energies, the electron current to an infinite planar probe for voltages negative with respect to the plasma potential is:

$$
i_{M}=A e N_{m} \sqrt{\frac{k T_{e}}{2 \pi M_{e}}} \exp \left(-\frac{e V}{k T_{e}}\right)
$$

where

$$
\begin{aligned}
A & =\text { area of probe } \\
e & =\text { electronic charge } \\
N_{m} & =\text { number of Maxwell electrons } \\
k & =\text { Boltzmann's constant } \\
T_{e} & =\text { electron temperature }
\end{aligned}
$$

$$
\begin{aligned}
M_{e} & =\text { mass of the electron } \\
V & =V_{s}-V_{0} \\
V_{s} & =\text { plasma potential } \\
V_{0} & =\text { potential applied to probe }
\end{aligned}
$$

When the probe is at plasma potential, $V=0$ and the saturation electron current $i_{s m}$ is

$$
i_{s m}=A e N_{m} \sqrt{\frac{k T_{e}}{2 \pi M_{e}}}
$$

From (1), the current-voltage curve will plot as a straight line on semilogarithmic graph paper, and the electron temperature can be determined from the slope of this line. The plasma potential can be found from the breakpoint in the current-voltage curve. Knowing the plasma potential and the electron temperature, the density of the Maxwell electrons can be found using (2).

Now consider the case of a monoenergetic group of electrons of number $N_{p}$ and energy $E_{p}$. Assume the velocities to possess a random distribution in space. The electron current to an infinite planar probe now is (Ref. 5):

$$
i_{p}=\frac{A e N_{p}}{4} \sqrt{\frac{2 e V_{p}}{M_{e}}}\left(1-\frac{V}{V_{p}}\right)
$$

where $V_{p}$ is the electron-volt energy equivalent of $E_{p}$. Thus, in this case, the current-voltage probe curves will be linear. Again, at plasma potential, when $V=0$ :

$$
i_{s p}=\frac{A e N_{p}}{4} \sqrt{\frac{2 e V_{p}}{M_{e}}}
$$

and since $V_{p}$ can be determined from the current-voltage curve, $N_{p}$ can be found from (4). Now, the total probe current $i$, when the two groups of electrons are present, is:

$$
i=i_{p}+i_{M}
$$

Since $V_{p}>k T_{e} / e$ when the arc voltage is large, for large negative voltages $V$

$$
i_{\mu}<<i_{p}
$$

so that $i$ is approximately linear. This linearity gives an indication of how to begin to apply these results to the probe curves.

Figure 10 shows a typical anomalous LPC on a linear scale. Note that, for large negative voltages, the curve 
can be approximated by a straight line corresponding to monoenergetic electrons of energy of approximately $22 \mathrm{v}$. When the straight line indicated is subtracted from the original data, the remaining curve plots as a straight line on a semilogarithmic plot. ${ }^{2}$ Figure 11 shows the original data on a semilogarithmic plot together with the remaining curve after the straight line has been subtracted. It is seen that the electron energy distribution leading to the anomalous LPC may be approximated by a distribution of electrons composed of two parts:

1. A "primary" distribution of monoenergetic electrons of high energy with randomly oriented velocities.

2. A Maxwell distribution of electrons with a low mean energy.

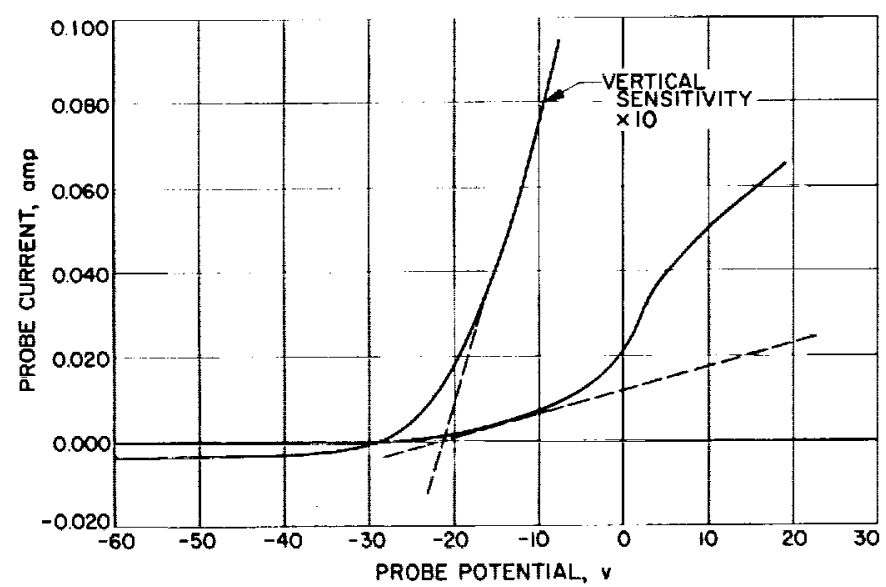

Fig. 10. Typical LPC from $x-y$ recorder (linear scale) arc voltage $-30 \mathrm{v}$ arc current-1.5 amp magnetic field-16 gauss (5 amp) flowrate-1.6 gm/hr

The above energy distribution is not an unreasonable distribution to assume for the electrons in the plasma. The primary electrons are those electrons from the cathode which are accelerated through the cathode sheath and undergo no collisions. The magnetic field of the engine will tend to randomly orient the directions of the velocity of the primary electrons. Maxwell electrons are

2Actually, one should analyze only the electron current to the probe. That is, use

$$
\boldsymbol{i}_{e}=\boldsymbol{i}+\left|\boldsymbol{i}_{+}\right|
$$

where $i_{e}$ is the electron current, $i$ is the probe current, and $i_{+}$is the saturated ion current. Since $\left|i_{+}\right|<<i$ for most of the voltage ranges considered, the probe current has always been used instead of the electron current for the analysis. produced from electron-electron collisions between the primary electrons which have undergone inelastic collisions with atoms and the electrons ejected from the atoms by the ionization process.

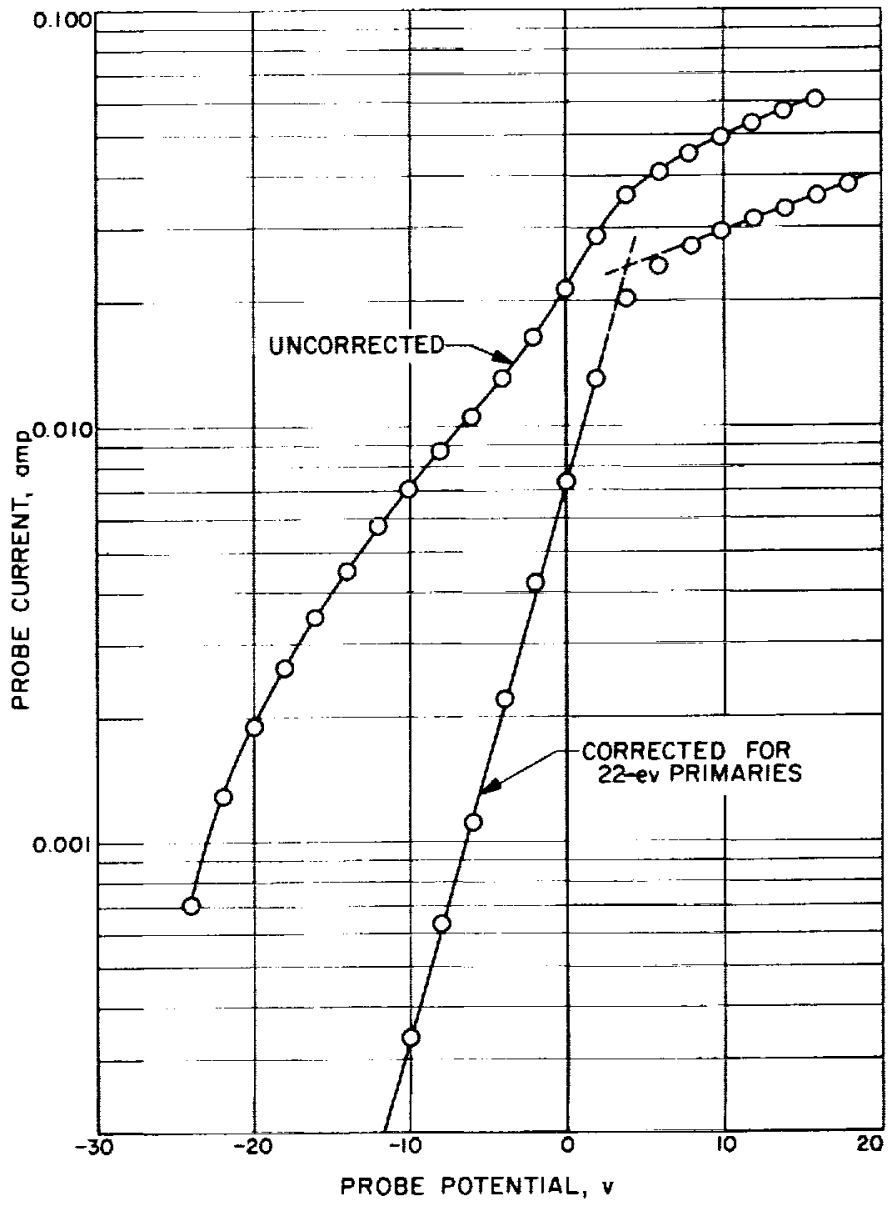

Fig. 11 . Corrected and uncorrected semilogarithmic LPC loperating conditions - same as for Fig. 10)

Electron-electron and electron-atom elastic collisions will tend to produce a spread in the energy of the primary distribution. One would expect the primary energy distribution to be better represented as gaussian or halfgaussian than as the monoenergetic spike used above. Thus, the current-voltage probe curves would not be exactly straight lines at large negative voltages, but a more complicated function, approximating a straight line as the gaussian becomes narrower.

All of the data taken were analyzed by using the monoenergetic-plus-Maxwell energy distribution approximation, even though in some cases the current-voltage curves were far from linear at negative probe voltages. 
It must be emphasized that the assumed distribution is only an approximation of the actual condition and is used at this time because of its simplicity in application. A better method would be to compute the energy distribu- tion directly from the LPC as was done by Druyvesteyn (Ref. 6) and Medicus, but this method involves taking second derivatives of the characteristics, which is a highly inaccurate and tedious procedure.

\section{RESULTS}

Figure 12 is a drawing of the positions at which measurements were made inside the engine. The axial coordinates are labeled 1 through 5 , while the radial coordinates are labeled $\mathrm{A}$ through $\mathrm{E}$.

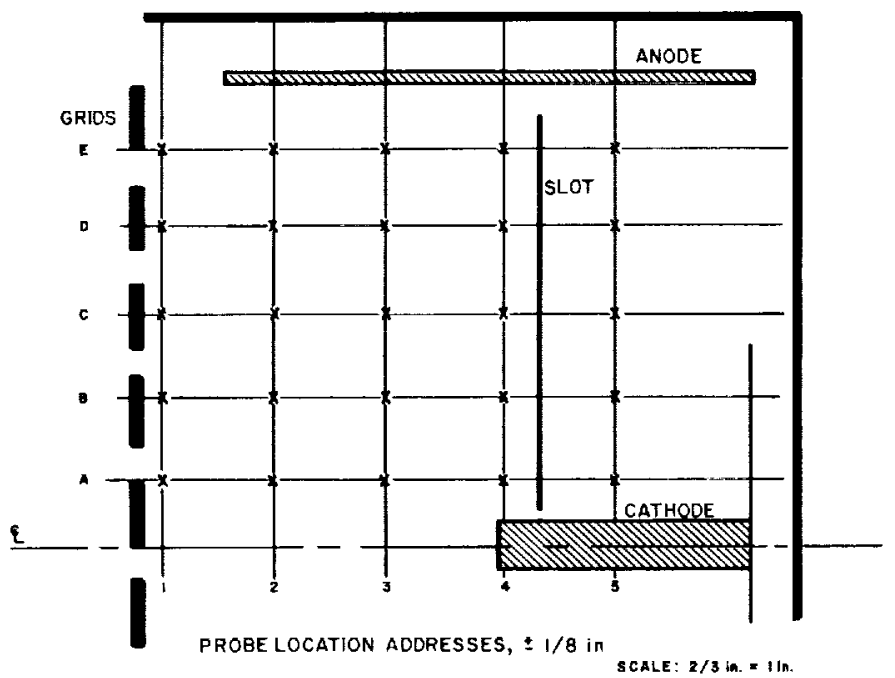

Fig. 12. Positions at which measurements were taken

Figures 13 through 20 present the results of applying the foregoing analysis to the LPCs. These graphs show the axial and radial plasma density and energy variations under various operating conditions. The graphs appear in pairs, the first being the no-beam condition, and the second being the corresponding data when a beam was being extracted. Therefore, a comparison of static and operating conditions can be made. All of the plasma properties obtained from the probe curves have been plotted except the plasma potential, which was very difficult to measure. The plasma potential usually varied between 2 to $7 \mathrm{v}$ above anode potential. The accuracy of these curves is estimated at only about $30 \%$, the large error being due mostly to the straight-line approximation to the primary electrons. Even so, the trends can be determined.

It is seen that the density of primary electrons drops off almost linearly with radial distance, while the Maxwell density usually drops much more slowly. There is also a definite drop in electron temperature in the radial direction. The drop in the electron temperature may be explained if there is significant energy exchange between the electrons of the primary and Maxwell groups. Since the primary and Maxwell densities are larger near the center of the engine, the greatest energy exchange between the groups would occur there; and since the energy of the primary electrons is higher than the Maxwell, the Maxwell mean energy would be raised.

The number density of ions was not measured, but if the plasma is assumed electrically neutral, the number of ions should equal the total number of electrons. This number also falls off with radial distance, most often following the density of Maxwell electrons, since the ratio of Maxwell to primary electrons is usually low. In fact, in a few cases (Fig. 15) the density of Maxwell electrons was found to increase going out from the cathode, approach a maximum about halfway between anode and cathode, and then fall off quite rapidly. The total number of electrons (and therefore the ions) were concentrated in an annular ring about the cathode. An annular ring of increased intensity could sometimes be observed in the blue glow from the end of the engine. 

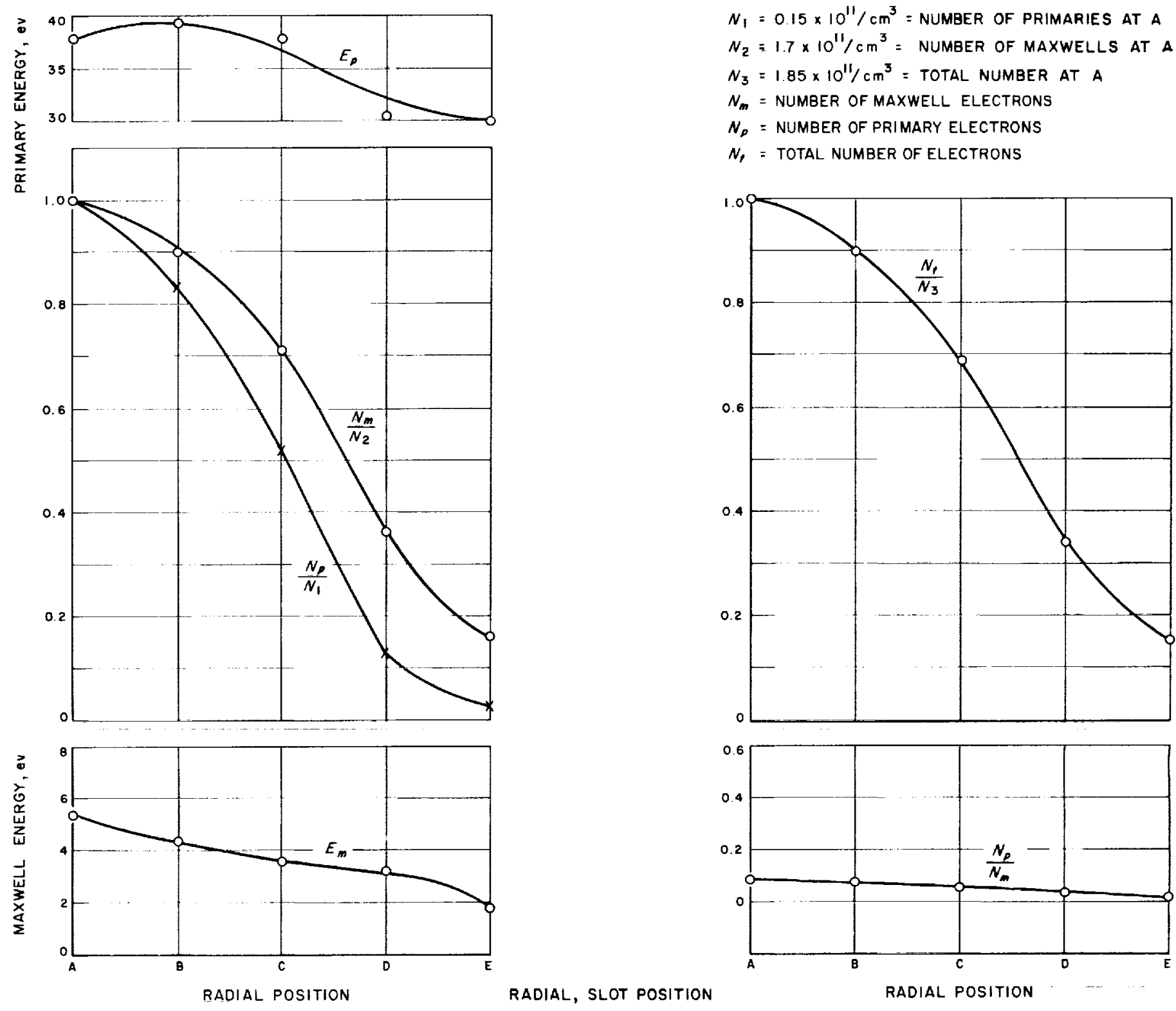

RADIAL, SLOT POSITION
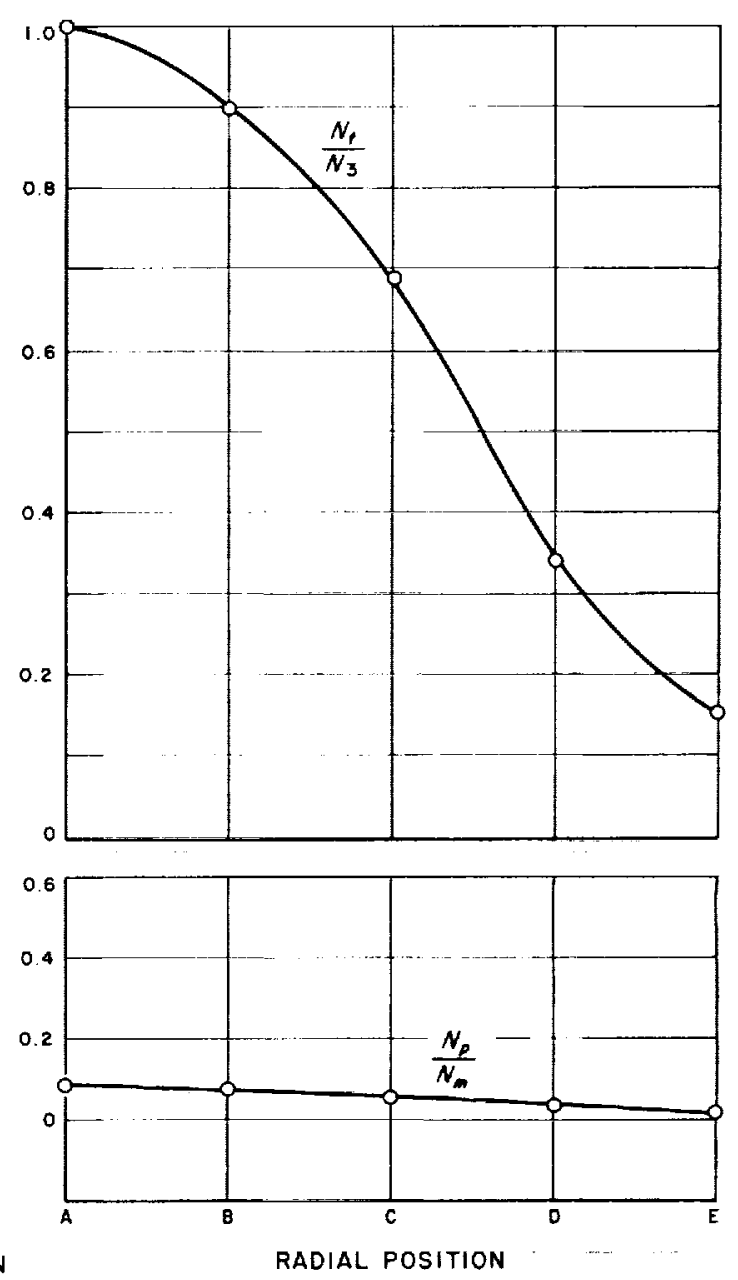

Fig. 13. Radial plot of electron density and energy variations (no accelerating potential applied) are voltage $-50 \mathrm{v}$ arc current-4 amp

magnetic field-20 gauss $(6 \mathrm{ampl}$

flowrate-1.5 gm/hr 

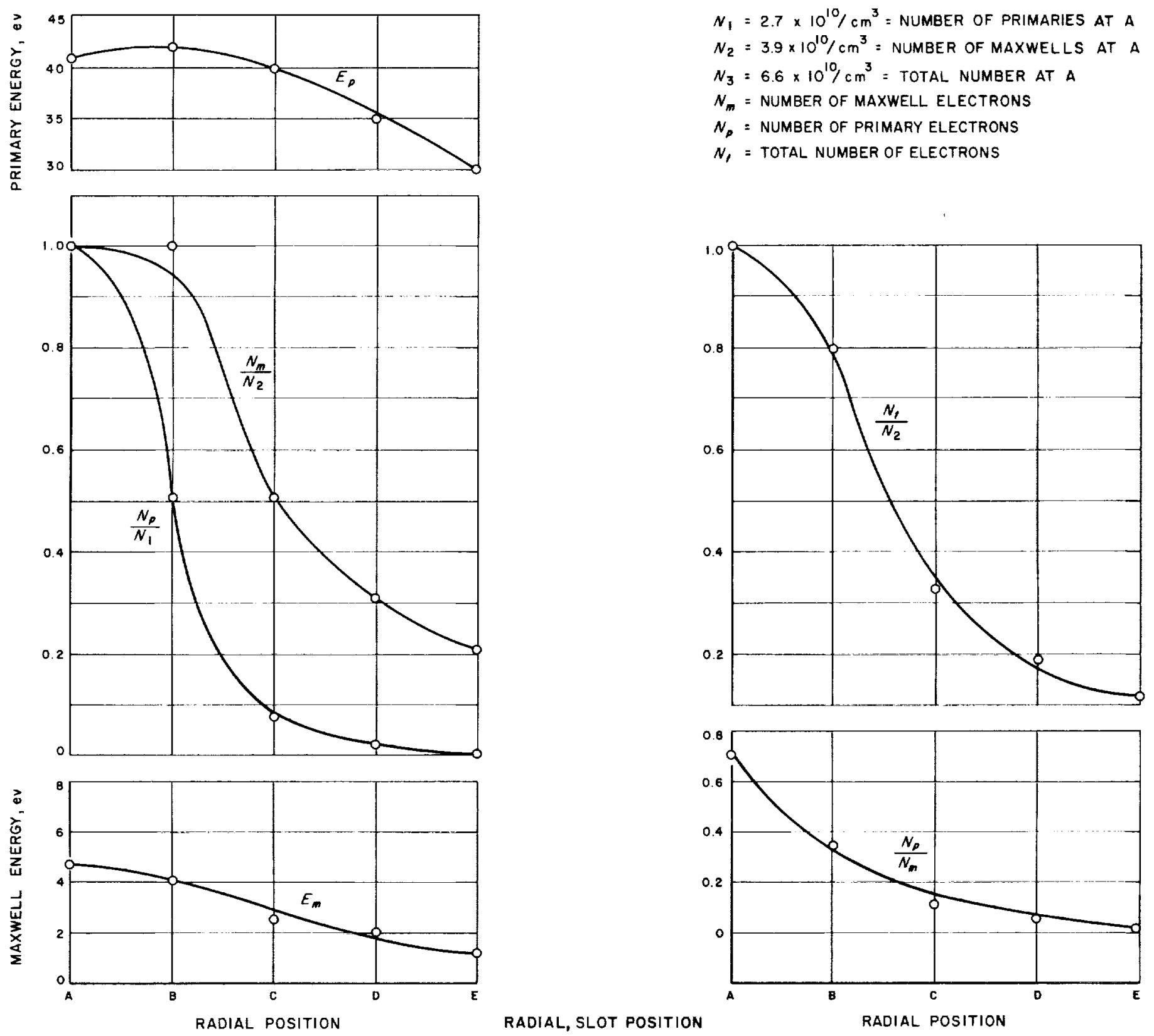

Fig. 14. Radial plof of electron density and energy variations (accelerating potential applied)

arc voltage $-50 \mathrm{v}$

arc current-4 amp

magnetic field-20 gauss (6 amp)

flowrafe- $1.5 \mathrm{gm} / \mathrm{hr}$ acceleration voltage-5 kv

beam current lengine)-160 ma

bias voltage-1 kv

interception current $-0.8 \mathrm{ma}$ 


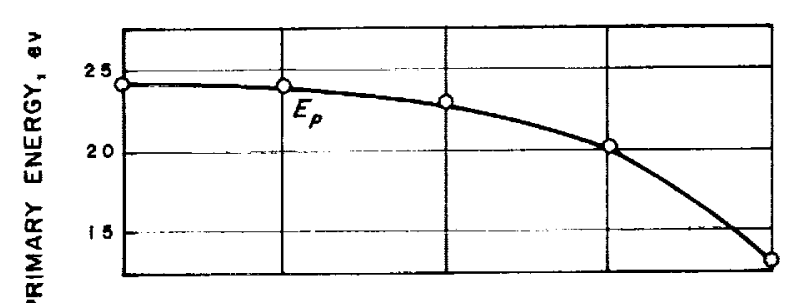

$N_{1}=0.223 \times 10^{10} \% \mathrm{~cm}^{3}=$ NUMBER OF PRIMARIES AT A

$N_{2}=2.52 \times 10 \% \mathrm{~cm}^{3}=$ NUMBER OF MAXWELLS AT A

$N_{3}=2.74 \times 10 \% \mathrm{~cm}^{3}=$ TOTAL NUMBER AT A

$N_{m}=$ NUMBER OF MAXWELL ELECTRONS

$N_{p}=$ NUMBER OF PRIMARY ELECTRONS

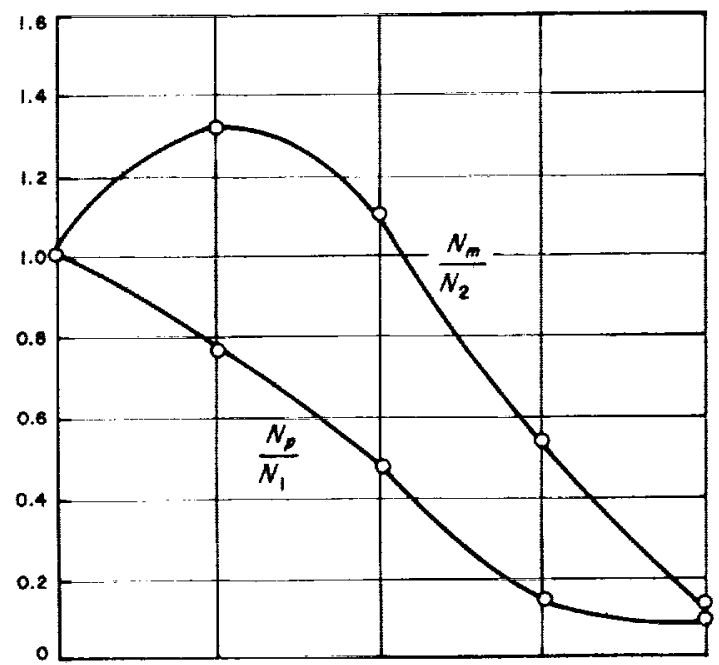

$N_{t}=$ TOTAL NUMBER OF ELECTRONS

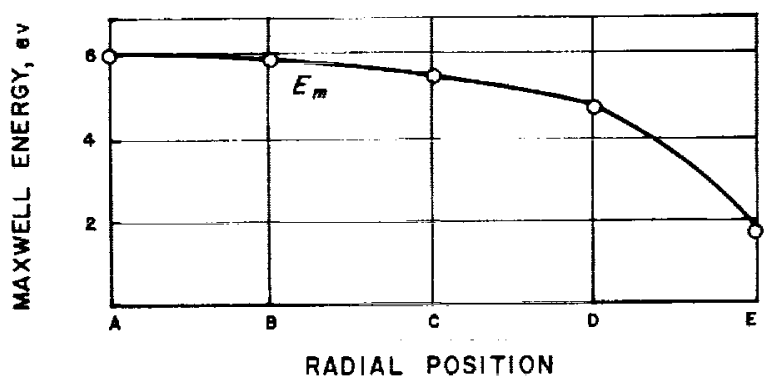

RADIAL, SLOT POSITION
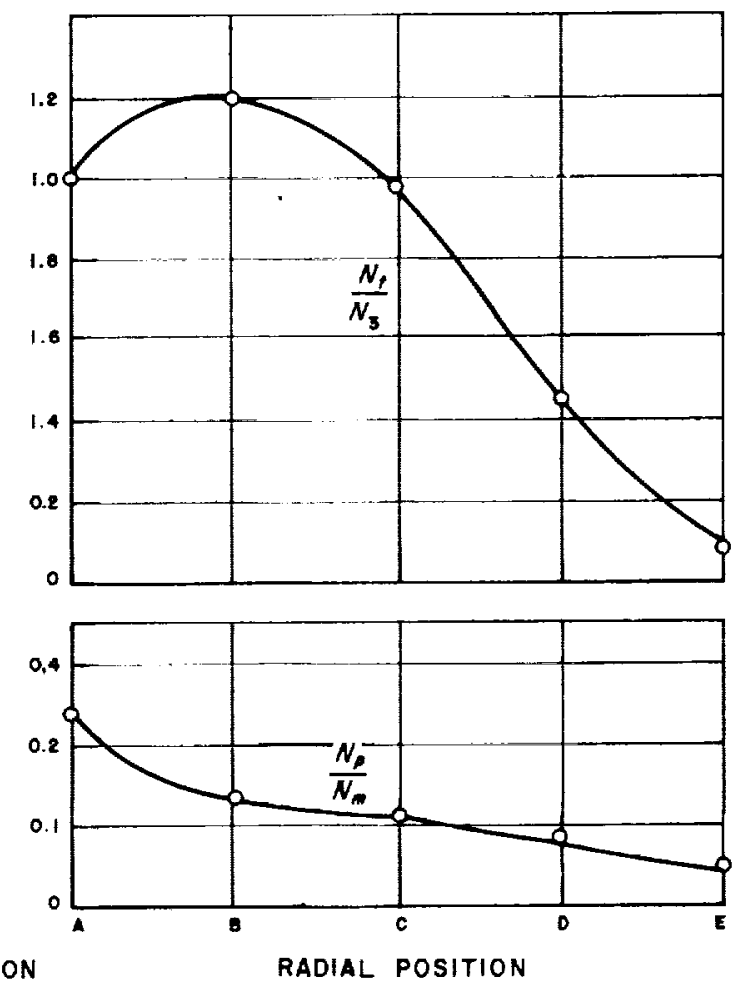

Fig. 15. Radial plot of electron density and energy variations (no accelerating potential applied)

are voltage-27 $v$ arc current- $0.9 \mathrm{amp}$ magnetic field-17 gauss (5.2 amp)

flowrate-1.4 gm/ hr 


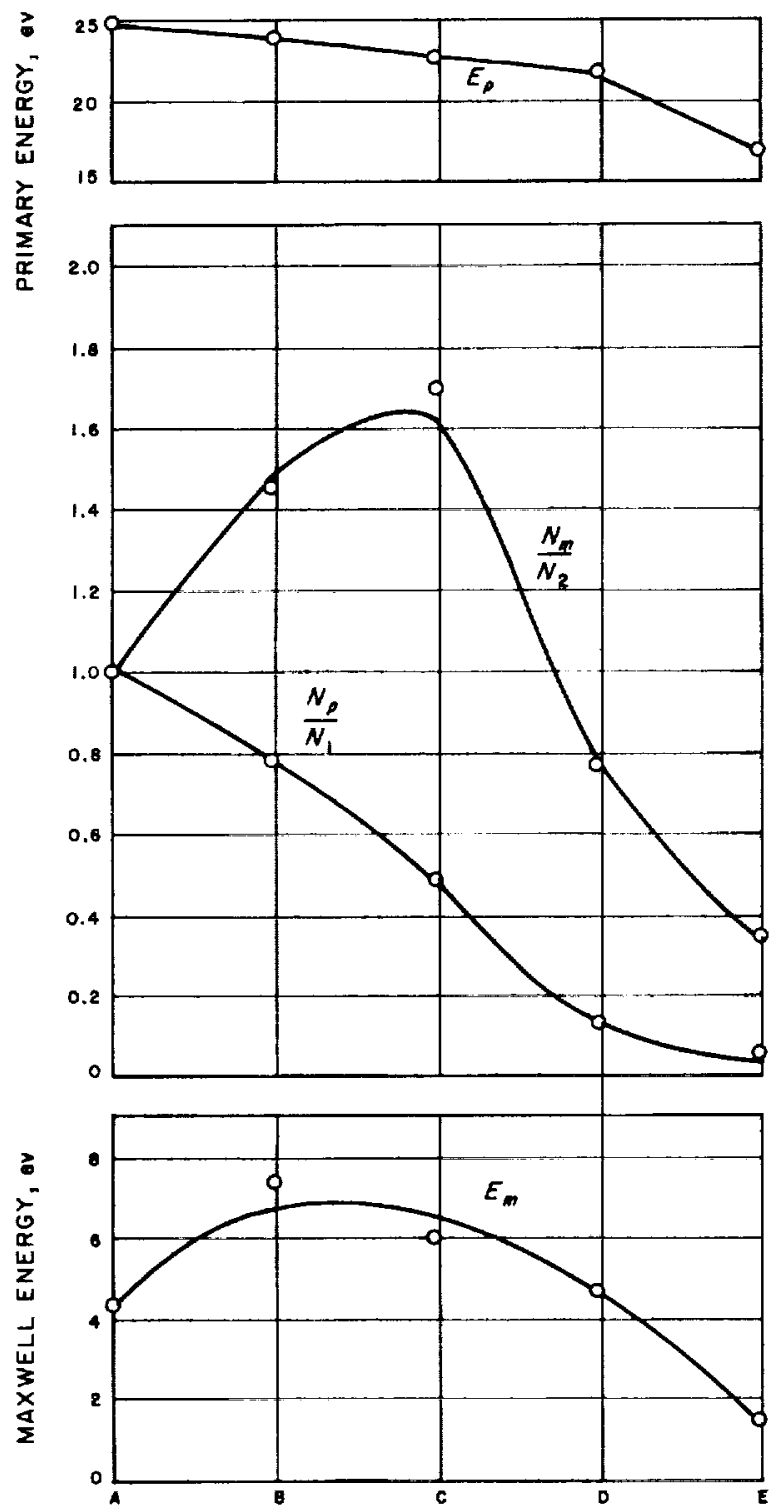

RADIAL POSITION
$N_{1}=1.15 \times 10^{10} / \mathrm{cm}^{3}=$ NUMBER OF PRIMARIES AT A

$N_{2}=0.409 \times 10^{10} / \mathrm{cm}^{3}=$ NUMBER OF MAXWELLS AT A

$N_{3}=1.56 \times 10^{10} / \mathrm{cm}^{3}=$ TOTAL NUMBER AT A

$N_{m}=$ NUMBER OF MAXWELL ELECTRONS

$N_{\rho}=$ NUMBER OF PRIMARY ELECTRONS

$N_{f}=$ TOTAL NUMBER OF ELECTRONS

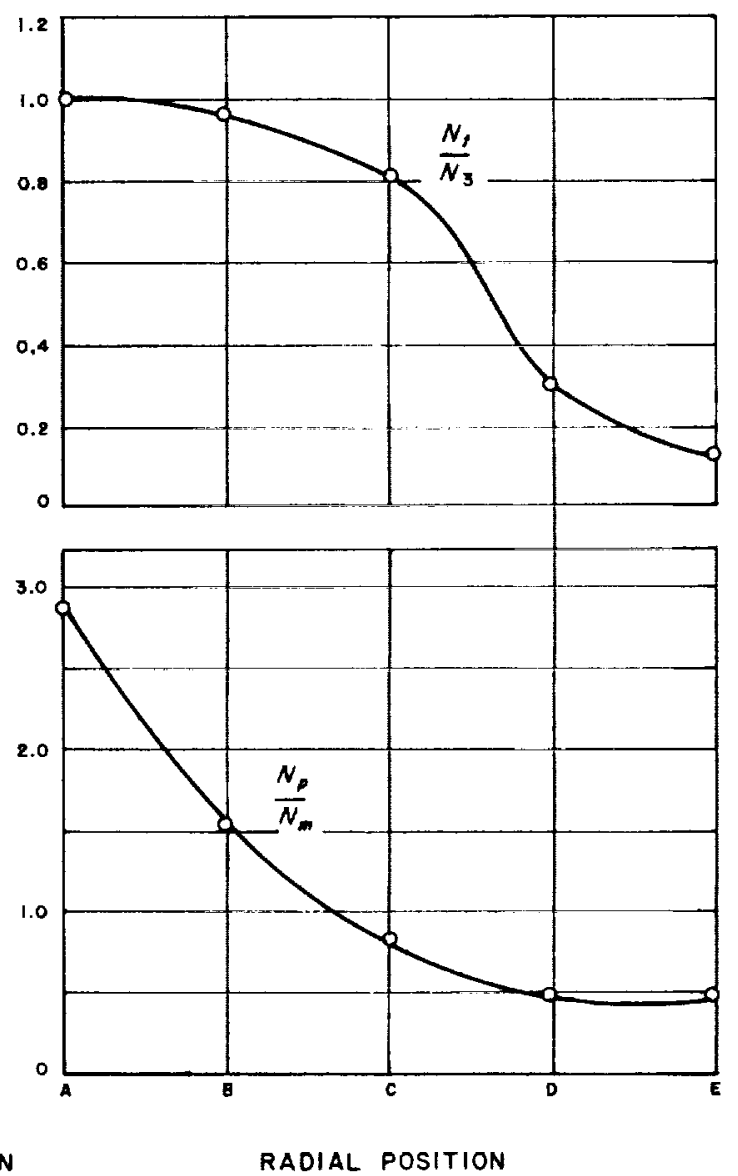

Fig. 16. Radial plot of electron density and energy variations (accelerating potential applied)

arc voltage $-28 \mathrm{v}$

arc current -0.85 amp

magnetic field-17 gauss (5.2 amp)

flowrate- $1.4 \mathrm{gm} / \mathrm{hr}$ acceleration voltage $4.9 \mathrm{kv}$

beam current lenginel-32 ma

bias voltage $-1 \mathrm{kv}$

interception current $-0.4 \mathrm{mo}$ 

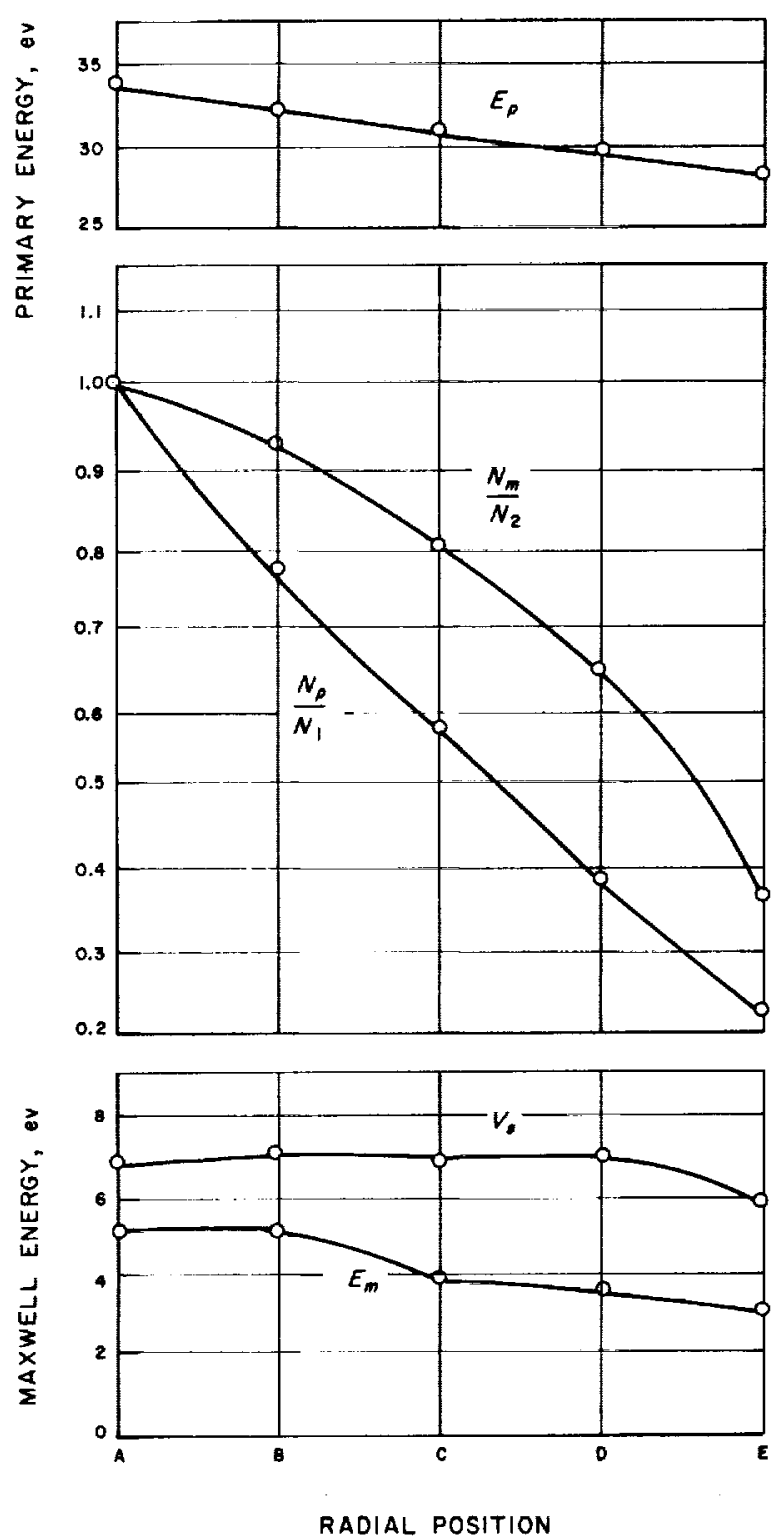

$N_{1}=0.195 \times 10^{11} / \mathrm{cm}^{3}=$ NUMBER OF PRIMARIES AT A $N_{2}=1.52 \times 10^{11} / \mathrm{cm}^{3}=$ NUMBER OF MAXWELLS AT A $N_{3}=1.72 \times 10^{11} / \mathrm{cm}^{3}=$ TOTAL NUMBER AT A $N_{m}=$ NUMBER OF MAXWELL ELECTRONS $N_{p}=$ NUMBER OF PRIMARY ELECTRONS $N_{i}=$ TOTAL NUMBER OF ELECTRONS
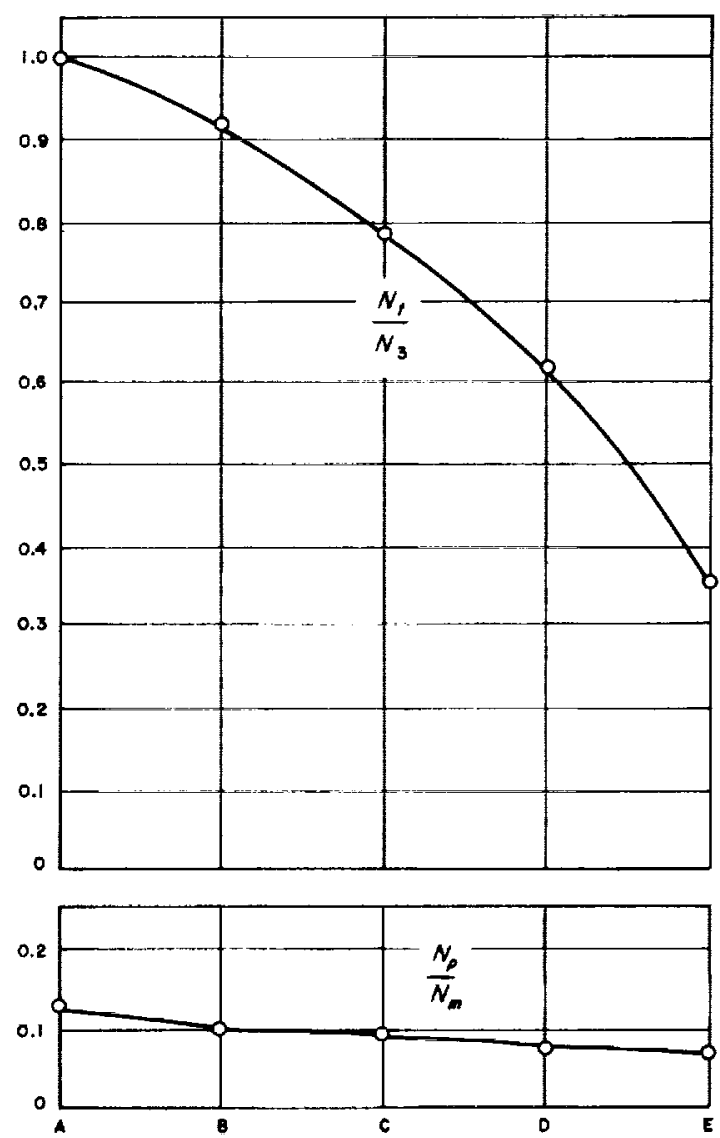

RADIAL POSITION

Fig. 17. Radial plot of electron energy and density variations near grids (no accelerating potential applied) (operating conditions-same as for Fig. 13) 

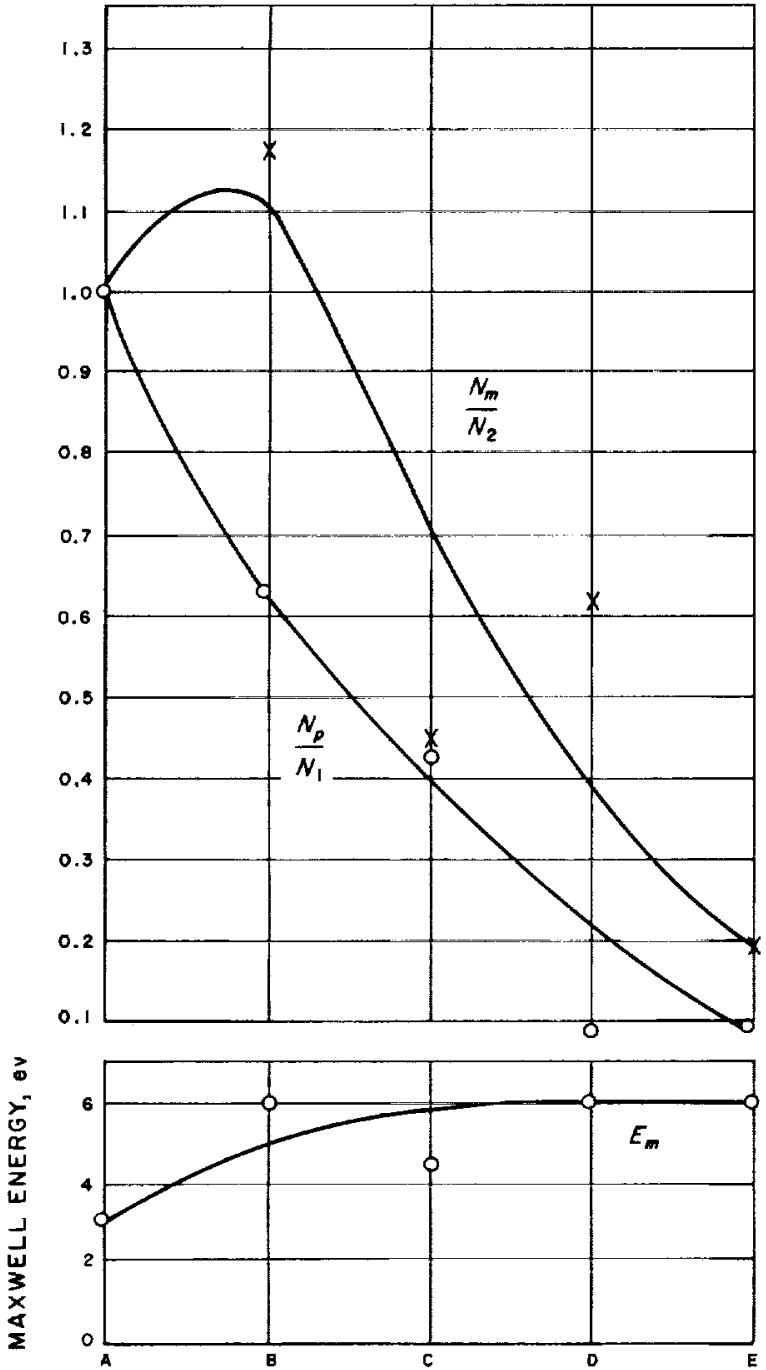

RADIAL POSITION
$N_{1}=0.508 \times 10^{10} / \mathrm{cm}^{3}=$ NUMBER OF PRIMARIES AT A

$N_{2}=2.52 \times 10^{10} / \mathrm{cm}^{\frac{3}{3}}=$ NUMBER OF MAXWELLS AT A

$N_{3}=3.03 \times 10^{10} / \mathrm{cm}^{3}=$ TOTAL NUMBER AT A

$N_{m}=$ NUMBER OF MAXWELL ELECTRONS

$N_{p}=$ NUMBER OF PRIMARY ELECTRONS

$N_{f}=$ TOTAL NUMBER OF ELECTRONS
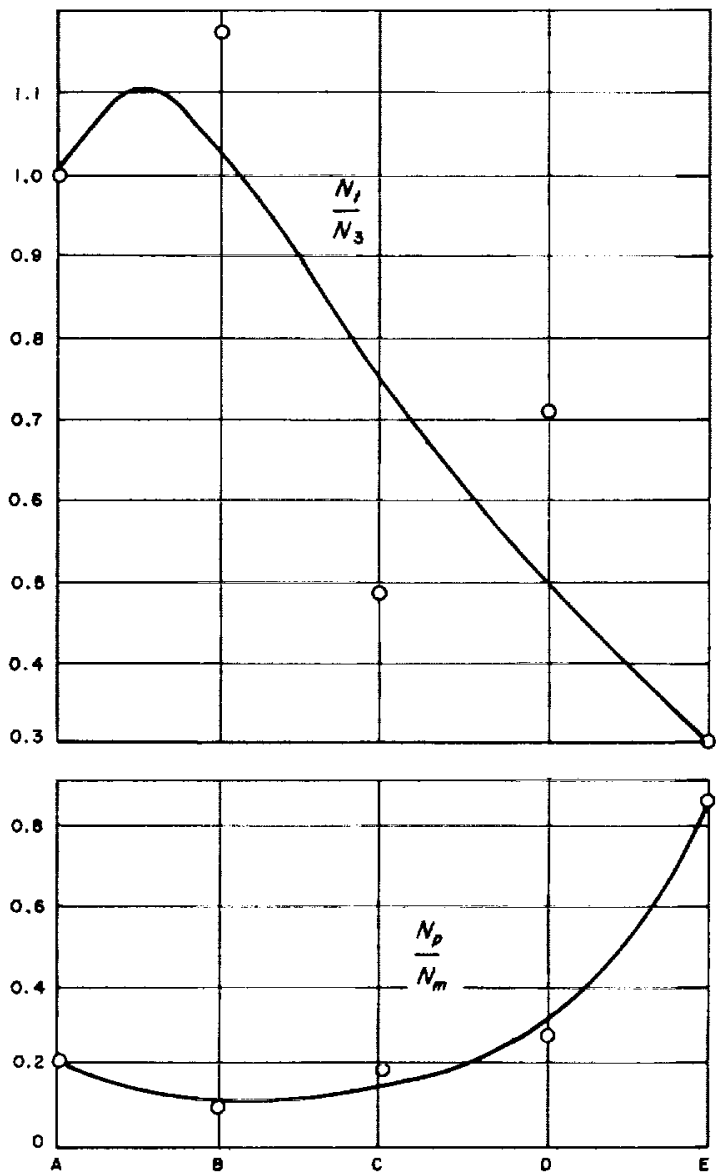

RADIAL, GRIDS

RAOIAL POSITION

Fig. 18. Radial plot of electron energy and density variations near grids (accelerating potential applied) (operating conditions_-same as for Fig. 14) 


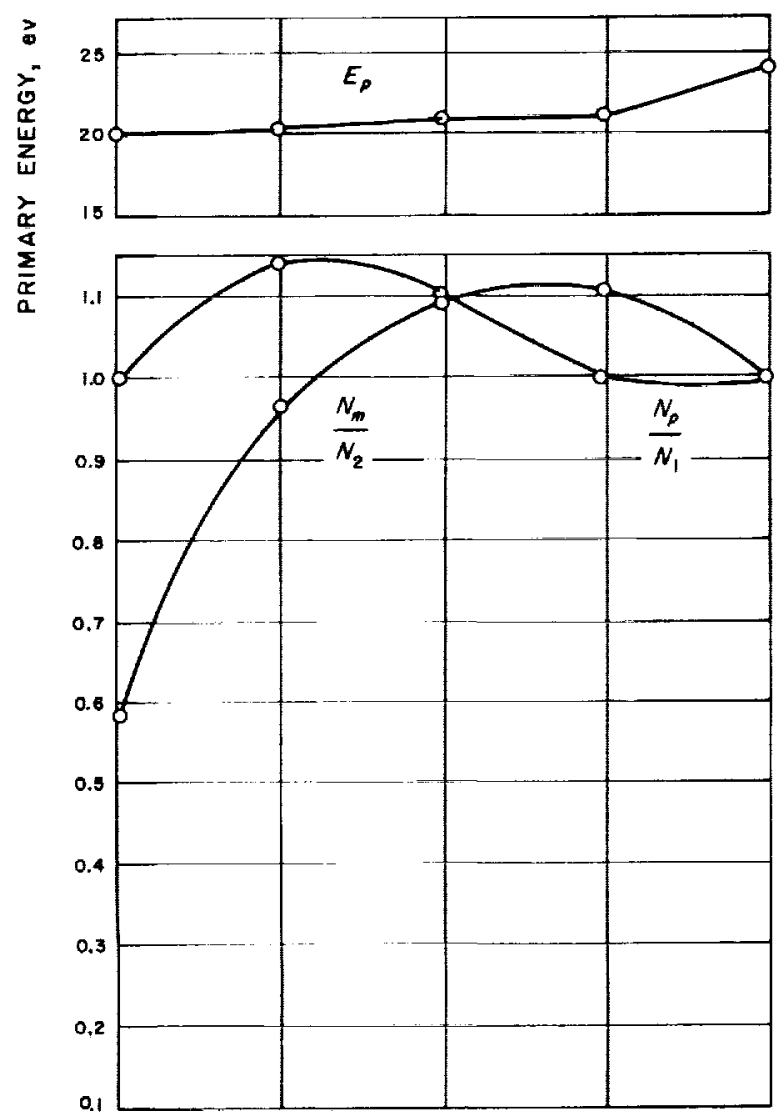

$N_{1}=1.09 \times 10^{10} / \mathrm{cm}^{3}=$ NUMBER OF PRIMARIES AT 5

$N_{2}=3.7 \times 10 \% \mathrm{~cm}^{3}=$ NUMBER OF MAXWELLS AT 5

$N_{3}=4.8 \times 1010 / \mathrm{cm}^{3}=$ TOTAL NUMBER AT 5

$N_{m}=$ NUMBER OF MAXWELL ELECTRONS

$N_{p}=$ NUMBER OF PRIMARY ELECTRONS

$N_{t}=$ TOTAL NUMBER OF ELECTRONS
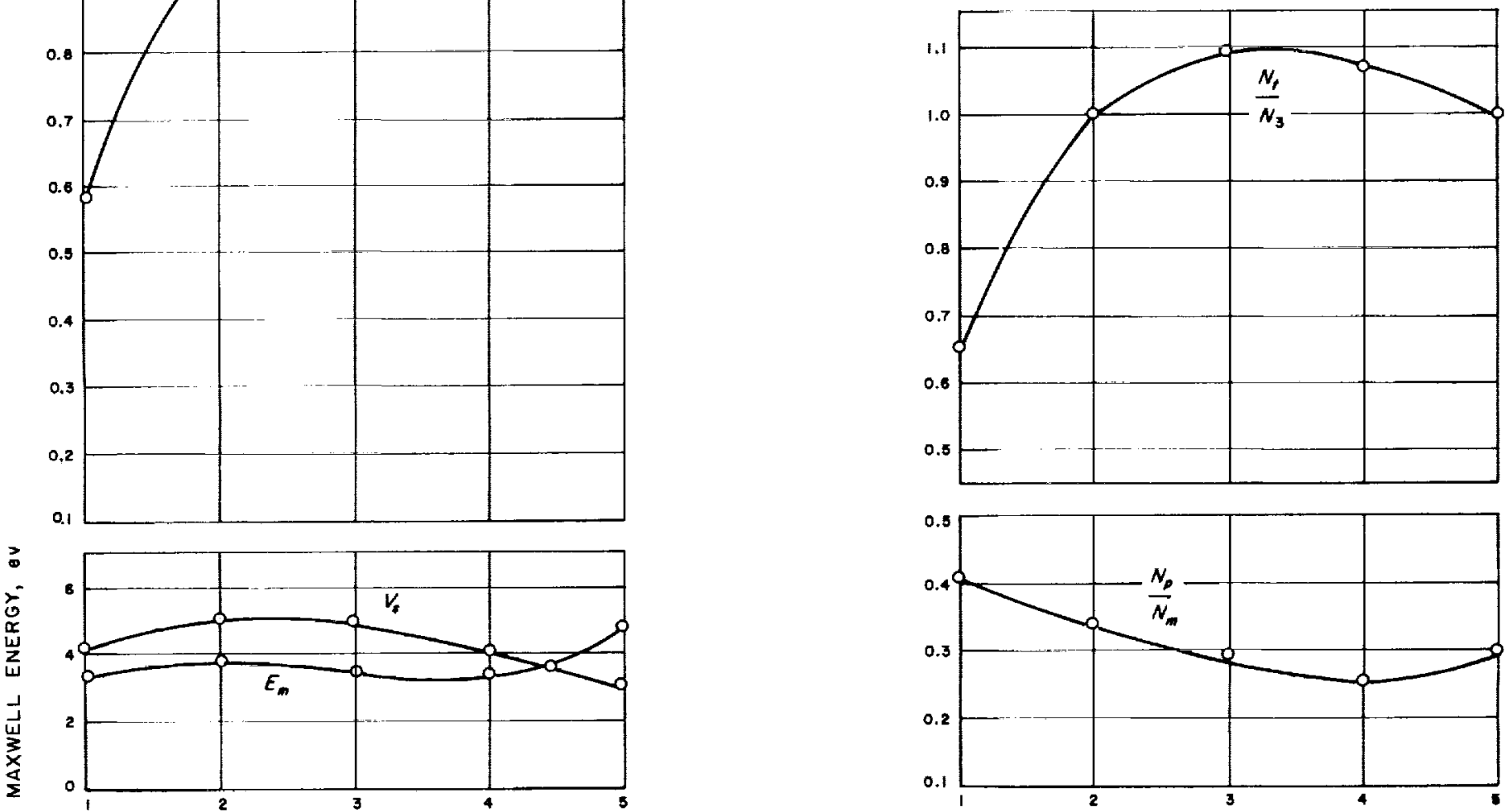

AXIAL POSITION

AXIAL, POSITION C

AXIAL POSITION

Fig. 19. Axial plot of electron energy and density variations (no accelerating potential applied) are volrage $-30 \mathrm{r}$ arc current-1.5 amp magnetic field-16 gauss ( 5 amp)

flowrate- $1.4 \mathrm{gm} / \mathrm{hr}$ 

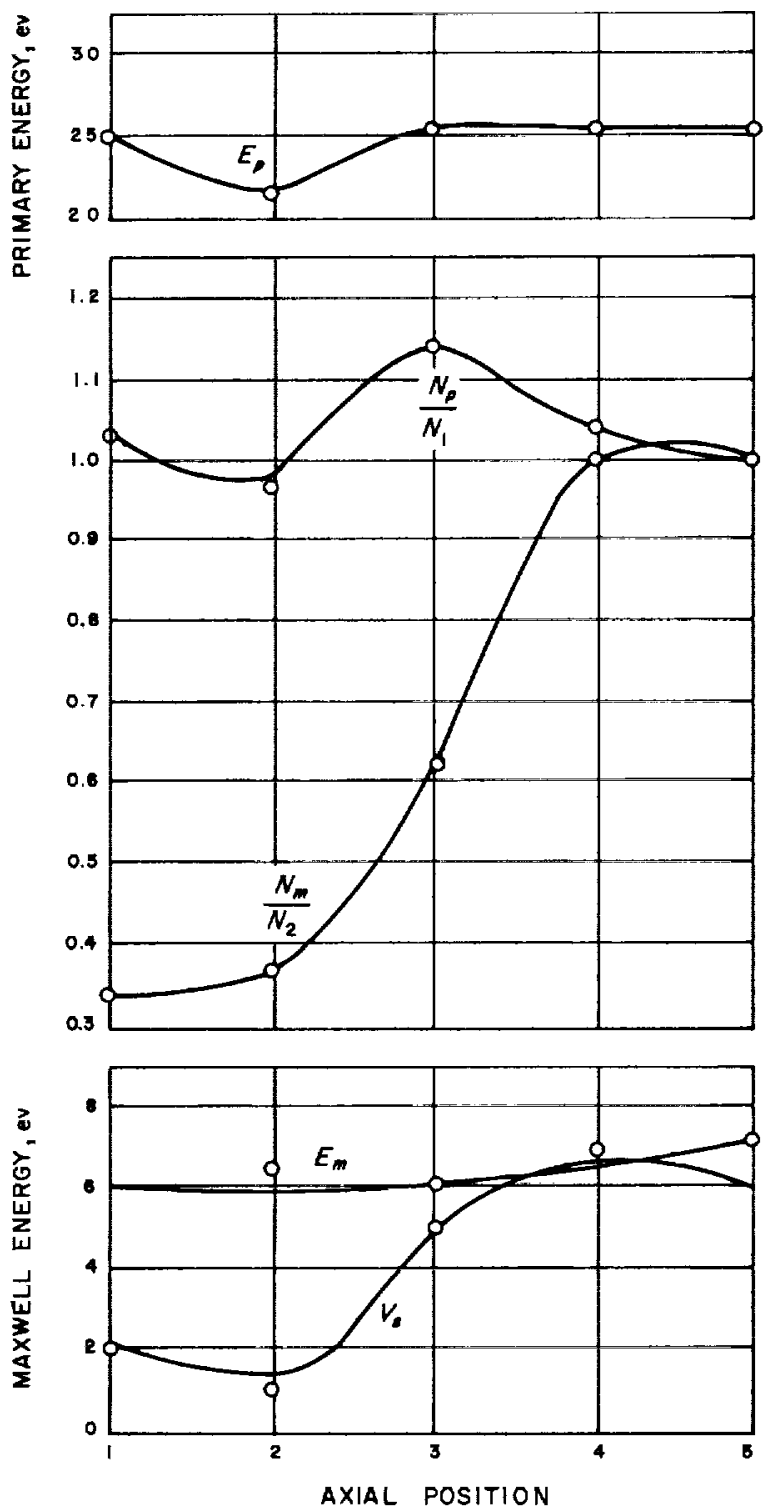

$N_{1}=1.17 \times 10^{10} / \mathrm{cm}^{3}=$ NUMBER OF PRIMARIES AT A $N_{2}=1.5 \times 10 \% \mathrm{~cm}^{3}=$ NUMBER OF MAXWELLS AT A

$N_{3}=2.67 \times 10^{10} / \mathrm{cm}^{3}=$ TOTAL NUMBER AT A

$N_{m}=$ NUMBER OF MAXWELL ELECTRONS

$N_{\rho}=$ NUMBER OF PRIMARY ELECTRONS

$N_{t}=$ TOTAL NUMBER OF ELECTRONS
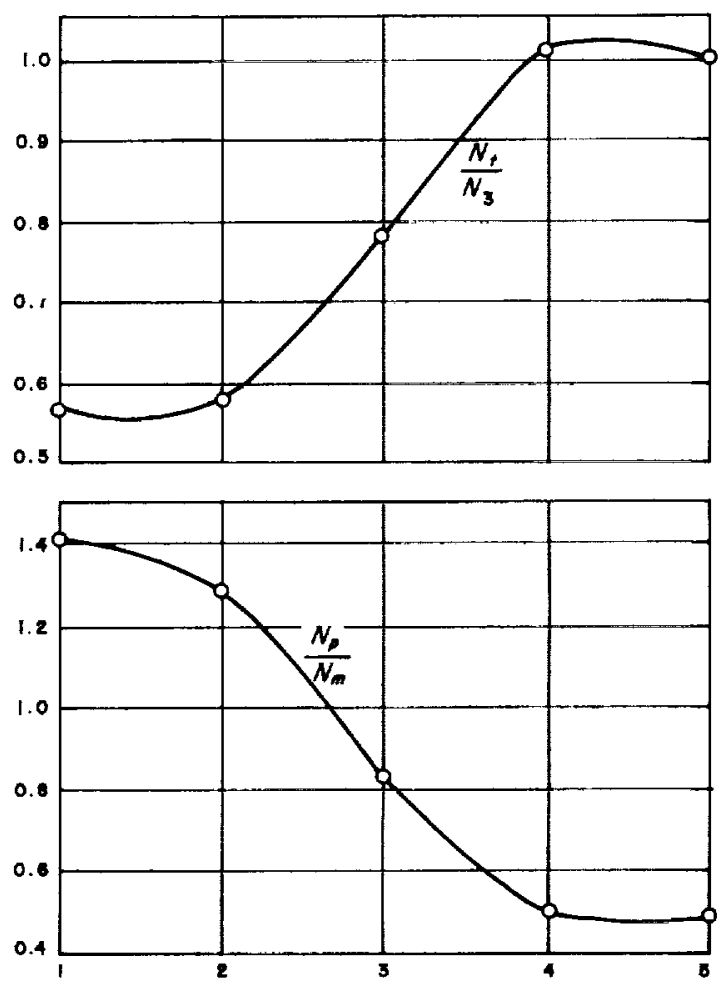

AXIAL, POSITION C

AXIAL POSITION

Fig. 20. Axial plot of electron energy and density variations (accelerating potential applied) are vollage-30 $\mathrm{v}$ arc current-0.9 amp magnetic field-16 gauss (5 amp) flowrate- $1.4 \mathrm{gm} / \mathrm{hr}$ acceleration voliage- $4 \mathrm{kv}$

beam current (engine) $-40 \mathrm{mo}$

bias voltage-1 kv

interception current- $0.4 \mathrm{ma}$ 
The effect of applying accelerating voltage is seen to reduce the density of Maxwell electrons (at the particular transverse position at which the radial traces were taken), which drastically increases the ratio of primary to Maxwell electrons. It is not clear why the accelerating potential should cause such an effect. One would expect that the plasma near the grids would shield the interior plasma quite effectively, but evidently this is not the case. The extraction of ions through the grids must set up axial electric fields which alter the spatial distribution of the lower-energy Maxwell electrons. The axial spatial distributions taken from axial sweeps of the Langmuir probe (Fig. 19, 20) do in fact show a gross rearrangement of the Maxwell electrons when high voltage is applied.

To see the effect of the primary electrons upon plasma formation, consider

$$
N_{+}(r, E)=\frac{d N_{+}(r, E)}{d t}
$$

the rate of production of positive ions at any point $r$ by electrons of energy $E$. This is given by

$$
\dot{N}_{+}(r, E)=N_{0}(r) f(E, r) \sigma_{i}(E) v
$$

where

$$
\begin{aligned}
N_{0}(r)= & \text { the number density of neutral atoms at the } \\
& \text { point } r \\
f(E, r)= & \text { the number of electrons with energy } E \text { at } \\
& \text { the point } r \text {, i.e., the spatial energy distribu- } \\
& \text { tion of the electrons } \\
\sigma_{i}(E)= & \text { the cross section for ionization of a neutral } \\
& \text { atom } \\
v= & \text { the velocity of the electron }
\end{aligned}
$$

Assume that the energy distribution of electrons is made up of two parts, a primary and a Maxwell part, so that $f$ can be written:

$$
f(E, r)=f_{p}(E) N_{p}(r)+f_{m}(E) N_{m}(r)
$$

where the subscript $p$ stands for primary and $m$ for Maxwell; $N_{p}$ and $N_{m}$ are the spatial number density distributions; and $f_{p}$ and $f_{m}$ are the energy distributions. For the primary energy distribution, assume a monoenergetic distribution at an energy $E_{p}$. Then,

$$
f_{p}(E)=\delta\left(E_{p}\right) \equiv \text { Dirac } \delta \text { function }
$$

The rate of ion production at point $r$ is determined by integrating (5) over all energies, using the energy distribution $(6),\left(6^{\prime}\right)$ and the usual Maxwell energy distribution:

$$
\begin{gathered}
\dot{N}_{+}(r)=\sqrt{\frac{2}{m}} \int_{0}^{\infty} N_{0}(r)\left[f_{p}(E) N_{p}(r)+f_{m}(E) N_{m}(r)\right] \\
\sigma_{i}(E) \sqrt{E} d E
\end{gathered}
$$

This yields on integration

$$
\dot{N}_{+}(r)=\sqrt{\frac{2}{m}} \sigma_{m} N_{1} N_{0}(r) N_{p n}(r) \sqrt{E_{p}}\left[Q_{p}(r)+Q_{m}(r)\right]
$$

where

$$
\begin{aligned}
m= & \text { electron mass } \\
\sigma_{m}= & \text { maximum value of the electron-mercury } \\
& \text { ionization cross section } \\
N_{1}= & N_{p} / N_{p n} \\
= & \text { maximum value of primary density } \\
N_{p n}(r)= & \text { normalized primary density } \\
E_{p}= & \text { primary energy } \\
Q_{p}(r)= & \sigma_{n}\left(E_{p}(r) / V_{i}\right) \\
= & \text { normalized ionization cross section of mer- } \\
& \text { cury; the approximation of } \sigma_{n} \text { used was } \\
& \text { developed by Kerrisk (Ref. } 2 \text { and } 7) \text { and is } \\
& \text { shown in Fig. } 21 .
\end{aligned}
$$

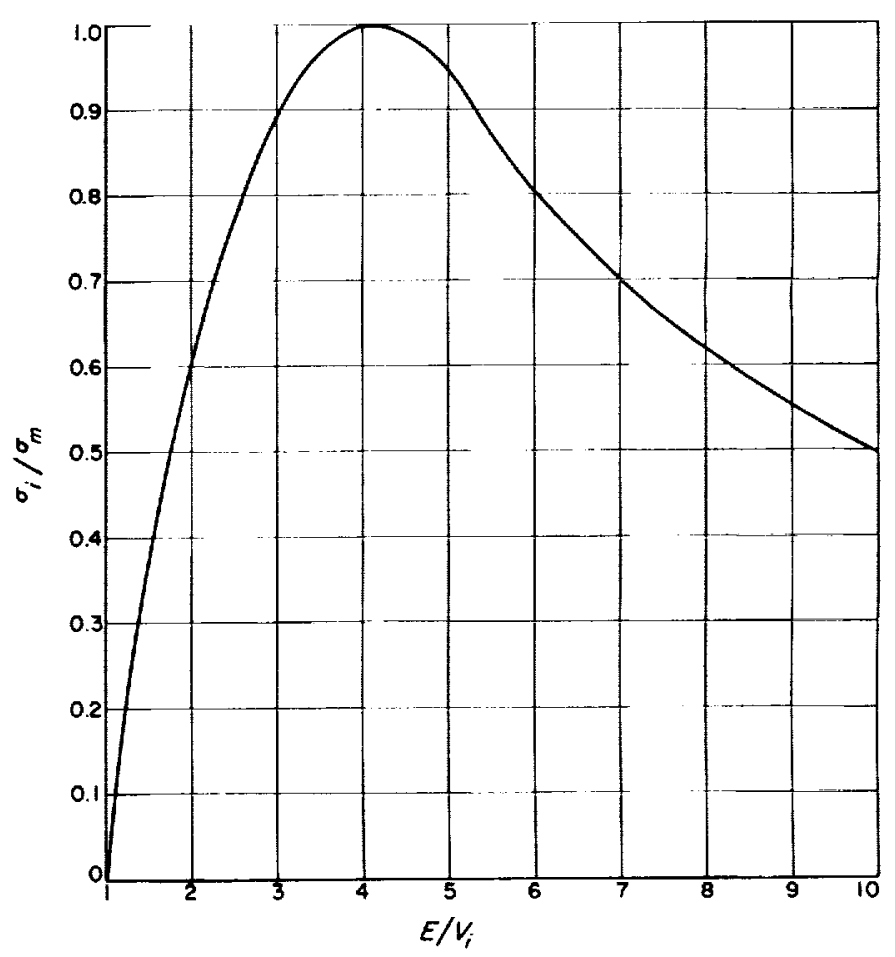

Fig. 21. Approximation of the mercury ionization cross section 


$$
\begin{aligned}
Q_{m}(r) & =\left(\frac{N_{m}}{N_{p}}\right)\left(\frac{E_{m}}{E_{p}}\right)^{1 / 2} \int_{0}^{\infty} f_{m}^{\sigma_{n}} \sqrt{E} d E \\
& =\left(\frac{N_{m}}{N_{p}}\right)\left(\frac{E_{m}}{E_{p}}\right)^{1 / 2} P_{m}\left(\frac{E_{m}}{V_{i}}\right)
\end{aligned}
$$

$P_{m}\left(\frac{E_{m}}{V_{i}}\right)=\begin{aligned} & \text { ionization coefficient of Maxwell elec- } \\ & \text { trons; this function has been evaluated }\end{aligned}$ by Kerrisk and is reproduced in Fig. 22

$E_{m}=$ mean Maxwell energy

$V_{i}=$ first ionization potential of mercury

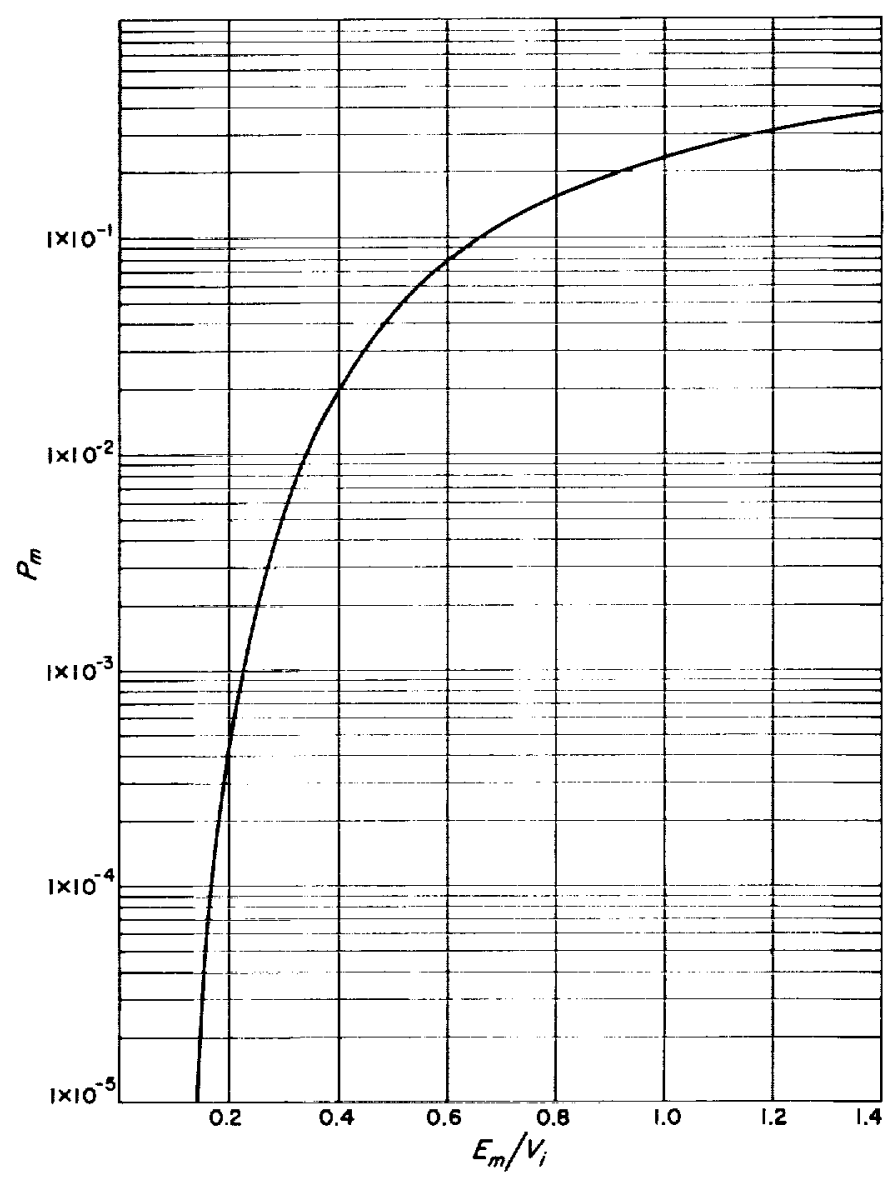

Fig. 22. Ionization coefficient for Maxwell electrons

Note that the functions $Q_{p}$ and $P_{m}$ will only have a radial variation if $E_{p}$ and $E_{m}$ vary radially. The functions $Q_{p}$ and $Q_{m}$ of Eq. (7) have been calculated from the spatial density distribution curves of Fig. 13 and 14 and are shown plotted in Fig. 23 and 24. One sees immediately that even though there are fewer primary than
Maxwell electrons, the function $Q_{m}(r)$ is at most $1 / 10$ as large as the function $Q_{p}(r)$. Furthermore, $Q_{m}$ is a sensitive function of the Maxwell mean energy and, since the mean energy always falls off in the radial direction, the ratio of $Q_{m}$ to $Q_{p}$ will be smaller at the anode than it is at the cathode. In other words, under most conditions, the term $Q_{m}$ in Eq. (7) can be neglected in comparison to $Q_{p}$. It follows that the primary electrons are more effective in producing ions.

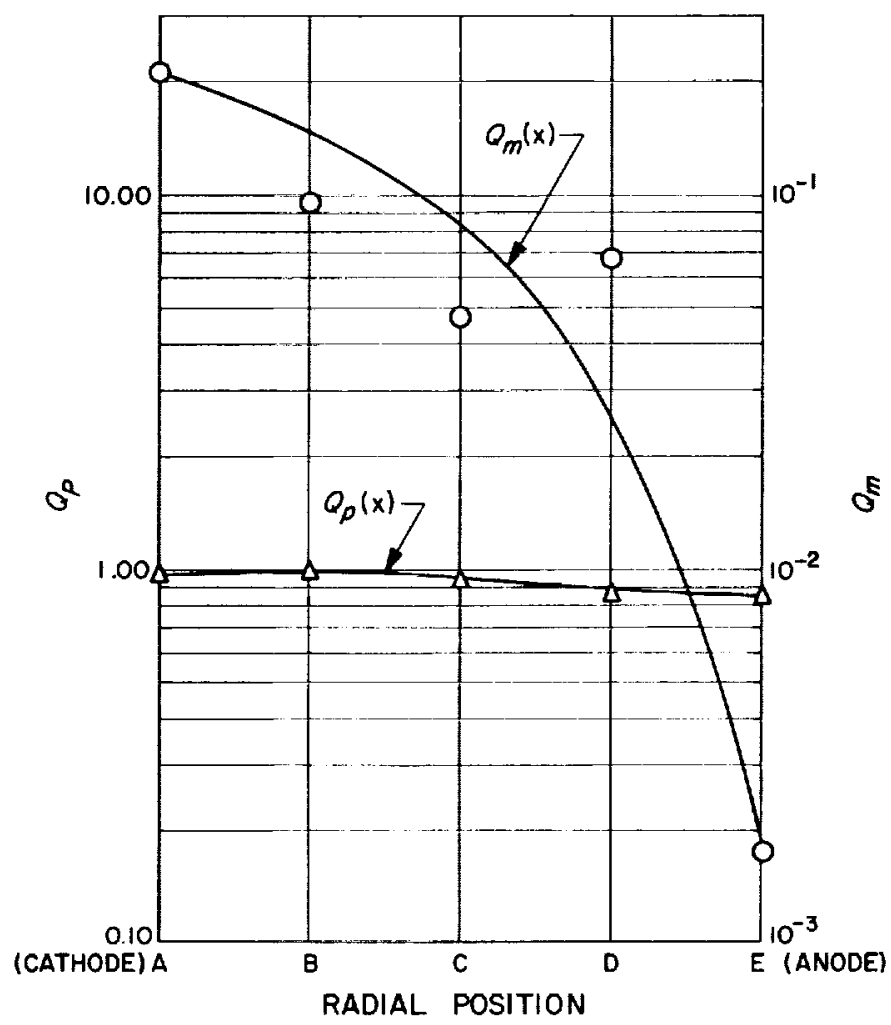

Fig. 23. Ionization rate curve (no accelerating potential applied)

loperating conditions-same as for Fig. (3)

The ion current density $J_{+}$necessary to form a stable sheath is given by Bohm (Ref. 8) to be

$$
J_{+}=e N_{+} \sqrt{\frac{k T_{e}}{M_{+}}}
$$

If it is assumed that this equation is valid in the extraction region of the engine near the grids, then from the measured values of the total ion density $\left(N_{+}\right)$and mean Maxwell energy $\left(T_{e}\right)$, the ion current can be calculated. The calculation has been performed on the probe data obtained when the engine was producing a 160 -ma beam 
(Fig. 17, 18) with agreement to the corrrect order of magnitude. At the grids the current density near the anode was about $1 / 4$ the current density near the center of the engine.

All of the data presented in this section are intended to be representative of a large amount of data under various operating conditions obtained from the probe curves. All the probe data for the following variations in engine parameters yield essentially similar results for the shape of the spatial density distributions.
Arc voltage

20 to $60 \mathrm{v}$

Arc current

Magnetic field

Cathode temperature

Propellant flowrate

Net acceleration voltage

Bias voltage

Beam current
0.85 to $4.7 \mathrm{amp}$

13 to 23 gauss

950 to $1250^{\circ} \mathrm{C}$

1.65 to $2.2 \mathrm{gm} / \mathrm{hr}$

3 to $8 \mathrm{kv}$

$\mathrm{l}$ to $1.5 \mathrm{kv}$

40 to $300 \mathrm{ma}$

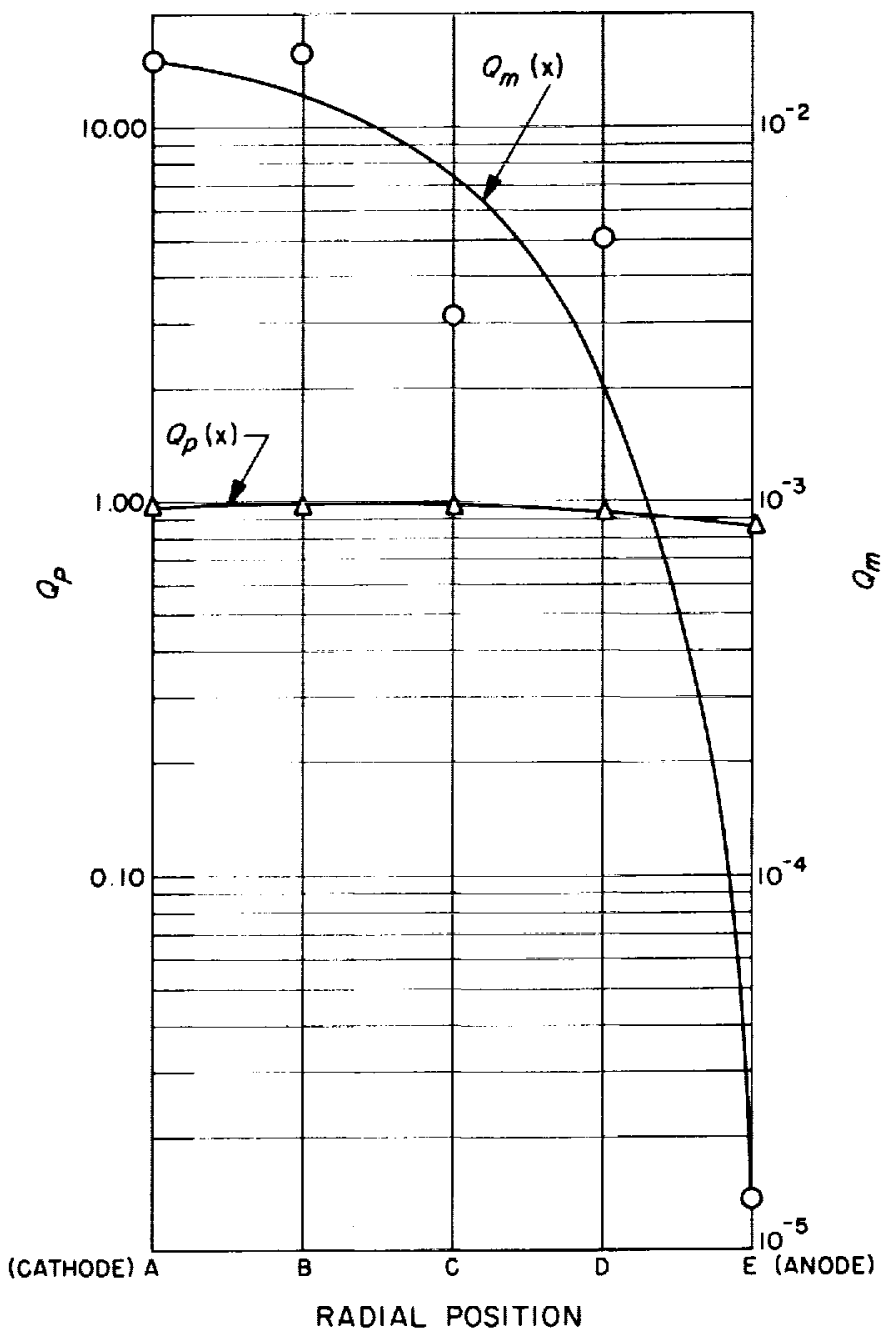

Fig. 24. Ionization rate curve laccelerating potential applied)

loperating conditions-same as for Fig. 14) 


\section{CONCLUSIONS}

Langmuir probe curves analyzed for the electron energy distribution for an operating (30 to 160-ma beam) and static (no beam, but plasma) Kaufman-type ion engine show that the energy distribution is not entirely Maxwellian. The energy distribution was found to consist of a high-energy primary distribution and a lower- energy Maxwell distribution. The primary electrons appear to be the main source of ion production. Further, the ion density near the grids as well as in the interior of the engine is not uniform. Any theoretical model of this source must include the primary distribution of electrons. 


\section{ACKNOWLEDGMENT}

The authors wish to acknowledge in particular the contributions of two of their colleagues: Mr. Roy W. Adams, who assisted in data collection, and Mr. Daniel J. Kerrisk, who contributed to illuminating discussions.

\section{REFERENCES}

1. Kaufman, H. R., An lon Rockef with an Electron-Bombardment Ion Source, NASA TN D-585, Washington, D. C., 1961.

2. Kerrisk, D. J., "Potentialities of Electron Bombardment lon Engines for Electric Propulsion,"' IRE Transactions on Space Electronics and Telemetry, SET-8, 1962, pp. 188-193.

3. Langmuir, I., and Mott-Smith, H. M., Gen. Elec. Rev., Vol. 27, 1924, pp. 449, 538, $616,762,810$.

4. Medicus, G., J. Appl. Phys., Vol. 29, June, 1958, p. 903.

5. Medicus, G., J. Appl. Phys., Vol. 27, October, 1956, p. 1242.

6. Druyvesteyn, M., Z. Physik, Vol. 64, 1930, p. 781.

7. Kerrisk, D. J., Arc-Type Ion Sources for Electrical Propulsion, TN 61-4, Aeronautical Systems Division, Wright-Patterson Air Force Base, May, 1961.

8. Bohm, D., Characteristics of Electrical Discharges in Magnetic Fields, edited by A. Guthrie and R. K. Wakerling, McGraw Hill Book Co., Inc., New York, N. Y., Ch. 3, 1949. 